\title{
3. Eine Systematik der Farbtheorien
}

Dieser Teil des Buches besteht aus drei Unterkapiteln. In dem ersten soll, anhand des Bikonditionals (BK), eine Unterscheidung zwischen dem Farb objektivismus und dem Farbsubjektivismus getroffen werden, die dann unter Einbeziehung der möglicherweise den Farben zugesprochenen Eigenschaftsarten für die Aufstellung einer systematischen Klassifikation der Theorien der Farben dienen kann. Das darauf folgende Kapitel wird diese Systematik mit der Intuition der Transparenz zu verbinden versuchen. Schließlich wird es, vor der konkreten Behandlung der einzelnen, ontologischen Positionen hinsichtlich von Farbeigenschaften, noch erforderlich sein, einen kurzen Blick auf die für eine Repräsentationalität der Farbwahrnehmungen unabdingbaren Normalbedingungen. Doch wichtiger ist sicherlich die Rolle der Transparenz innerhalb einer Analyse der Natur der Farben. Und diese kann nur dann sinnvoll diskutiert werden, wenn die beiden Hauptalternativen - der Subjektivismus und der Objektivismus - angemessen differenziert worden sind: was im folgenden geschehen soll.

\subsection{Subjektivismus und Objektivismus}

\subsubsection{Subjektrelativität und Subjektabhängigkeit}

In diesem Kapitel soll die Möglichkeit diskutiert werden, wie eine Grenze zwischen einem Subjektivismus und einem Objektivismus eindeutig gezogen werden kann. Dabei soll die These vertreten werden, daß ontologische Subjektabhängigkeit im Grunde nichts anderes ist als ontologische Geistabhängigkeit. So ist diese Einsicht auch bereits bei der Definition des Objektivitätsmerkmales des repräsentationalen Aspektes angewandt worden ist. Eine vergleichbarre Bestimmung der subjektivistischen Position hinsichtlich von Farben findet sich bei Keith Campbell:

„At least one important necessary condition of any subjectivist account of colour seems to me to be this: A subjectivist thesis must have as a consequence that, in the absence of perceivers with colour vision, there are no colours. Subjectivism 
requires that sunsets have not been red since time immemorial, but only since the appropriate living forms evolved.“ (K. Campbell (1993): 264)

Diese Bedingung ist erfüllt, wenn Farbtatsachen auf die eine oder andere Weise von Farbwahrnehmungszuständen bestimmter Subjekte ontologisch abhängen werden. Dies wird somit die Grundthese der Farbsubjektivisten darstellen müssen. Doch sie kann, wie sich zeigen wird, in verschiedener Stärke interpretiert werden. Die schwächste Form stellt aller Voraussicht nach die dispositionalistische Analyse dar, derzufolge es für subjektive Farbtatsachen nur faktisch möglich sein muß, mittels Farbwahrnehmungen gesehen zu werden; subjektive Farbtatsachen haben demnach auch dann Bestand, wenn sie nicht gerade wahrgenommen werden. Aber gerade bei der dispositionalistischen Position wird es die Möglichkeit geben, zwischen zwei alternativen Positionen zu wählen: zum einen können Farbtatsachen vom Auftreten mentaler (und womöglich zusätzlich phänomenaler) Zustände abhängig sein, zum anderen hingegen bloß vom Vorkommen bestimmter Zustände im Gehirn oder dem visuellen Sinn. Es ist breiter Konsens, daß eine ontologische Subjektabhängigkeit nur dann vorliegt, wenn es sich bei den betreffenden Zuständen um mentale Zustände handelt. So nehmen die einzelnen, weiter unten erwähnten Definitionen des Farbsubjektivismus alle explizit Bezug auf Farbwahrnehmungen von Subjekten. Ontologische Geistabhängigkeit - oder Subjektivität - ist damit nichts anderes als ontologische Subjektabhängigkeit.

Um diese Behauptung plausibel zu machen, lohnen sich folgende Überlegungen. Von Subjekten (in einem ganz basalen Sinn) wird üblicherweise immer dann gesprochen, wenn ein bewußtes Lebewesen mit einem Geist gemeint ist. Daß die Grenze zwischen Lebewesen mit und Lebewesen ohne mentale Zustände nicht eindeutig gezogen werden kann, braucht uns hier nicht weiter zu stören. Subjekte finden ihren Platz in der materiellen Welt durch die physikalischen Eigenschaften ihres Körpers und ihres Gehirnes, von denen ein Teil für die Realisierung des Geistes verantwortlich gemacht wird. Doch ganz unabhängig davon, auf welche Weise das Körper-GeistProblem gelöst werden kann, gelten Subjekte als Subjekte allein aufgrund ihrer Mentalität. Es läßt sich somit in gewissem Sinne sagen, daß den Subjekten ganz allgemein ein Geist wesentlich zukommt, während ihre physikalische Beschaffenheit ihnen eher akzidentiell ist. Ein Mensch oder ein 
Tier zählt nicht deswegen als ein bewußtes Subjekt mit einem (eingeschränkten) eigenen, unabhängigen, mentalen Leben, weil es eine bestimmte physikalische oder biologische Konstitution aufweist, ja streng genommen nicht einmal, weil es ein Gehirn besitzt und bestimmte Verhaltensmuster zeigt; sondern letztlich allein deswegen, weil es über einen Geist verfügt. Es scheint sogar bis zu einem gewissen Grad denkbar zu sein, daß es eventuell unter ganz anderen Umständen auch Subjekte geben könnte, die nur aus einem Geist ohne Körper beständen - und immer noch Subjekte wären.

Nun könnte man sich vor diesem Hintergrund zwei Grade von Subjektabhängigkeit vorstellen: einerseits könnten bestimmte Tatsachen von der Existenz mentaler Zustände - als Reaktionen der Subjekte - abhängen, andererseits von bloß neuronalen oder cerebralen (Farben können zum Beispiel sowohl als Dispositionen in Hinsicht auf Farbwahrnehmungen als auch in Hinsicht auf Zustände des visuellen Systemes angesehen werden). Sind die in Frage stehenden Tatsachen ontologisch an Zustände gebunden, die mit metaphysischer Notwendigkeit Bestandteil eines Subjektes sind, das heißt also: an mentale Zustände, dann handelt es sich wirklich um eine ontologische Subjekt- oder Geist-Abhängigkeit, im Gegensatz zu einem objektiven Status. Dies ist bei Farbwahrnehmungen als Reaktionen der Fall. Besteht die Abhängigkeit jedoch nur zu Subjekten kontingenterweise zukommenden Zuständen, dann liegt eine ontologisch irrelevante Subjektrelativität vor: wie etwa bei Reizungen bestimmter Nervenzustände im visuellen System als Reaktionen. Letzteres gilt nicht unbedingt für alle cerebralen Zustände eines Subjektes: diejenigen, die mit mentalen Zuständen in einer Realisierungs- oder sogar Identitätsrelation stehen, sollten vorsichtshalber ausgeschlossen werden, da sie nach einigen Theorien des Geistes vielleicht eine gewisse ontologische Abhängigkeit von mentalen Zuständen aufweisen.

\subsubsection{Der Euthypron-Kontrast}

Der Begriff der sekundären Qualität wird üblicherweise nur auf direkt sinnlich wahrnehmbare Eigenschaften angewandt. Diese Tradition leitet sich unter anderem von Locke her, der allein Farben, Klänge, Gerüche und 
Geschmacke als sensible qualities bezeichnet (vgl. Locke (1706): Book II, Kap. 8, § 23). Die Hauptfrage, die sich dabei in der Diskussion stellt, ist, inwiefern die mentalen Wahrnehmungszustände für die Existenz der entsprechenden Tatsachen konstitutiv sind. Doch es gibt auch andere Klassen von Eigenschaften, hinsichtlich derer vergleichbare Diskurse geführt werden können, obwohl hier nicht unmittelbare Sinneserfahrungen, sondern kognitiv höhere und komplexere mentale Zustände in Frage kommen. Als klassische Beispiele können ästhetische und moralische, aber auch mathematische und semantische Eigenschaften angeführt werden (vgl. Wright (1988); (1993); (1998); Boghossian (1989)). Setzt man einen globalen Realismus als gegeben voraus, dann geht es in allen Fällen - ob sekundären Qualitäten oder allein begrifflich fixierbaren Eigenschaften - um die Frage, ob ein lokaler Realismus hinsichtlich der betreffenden Klasse von Eigenschaften vorliegt, oder nicht. Die Antwort auf diese Frage entscheidet darüber, ob die diskutierten Merkmale wirklich Bestandteil der Realität sind, oder nur von unserem Geist dieser Welt auferlegt werden.

Zur Diskussion steht damit die Entscheidung für eine objektivistische (realistische) oder eine subjektivistische (anti-realistische) Theorie über die fraglichen Charakteristika. Zudem gibt es noch die (irrealistische) Option, die Instantiiertheit der zur Debatte stehenden Eigenschaften ganz zu leugnen; doch üblicherweise wird diese Position als eine Variante des Subjektivismus angesehen. Wie schon zu Anfang der Abschnitt über die empiristische Tradition gezeigt hat, beschäftig sich die Diskussion darüber, die sekundären Qualitäten wirklich von den sogenannten primären Eigenschaften verschieden sind, im Grunde mit nichts anderem als der Frage, wie die sekundären Qualitäten in ein naturwissenschaftliches Weltbild eingebunden werden können. Der Rest des Kapitels Abschnitt soll nun einen Leitfaden für die Beantwortung dieser Frage bereitstellen, indem die Differenzierung der objektivistischen Alternative von der subjektivistischen noch klarer gefaßt und auf dieser Grundlage schließlich eine systematische Klassifikation der Theorien der Farben aufgestellt werden soll. Die Ausgangsbasis für diese Klassifikation wird vor allem das Bikonditional (BK) verkörpern. Dabei sollen die Überlegungen von Crispin Wright zu diesem Thema als ständige Orientierungshilfen dienen. Ein berühmtes Beispiel für den Disput zwischen Objektivisten und Subjektivisten (oder auch ontologischen Realisten und Antirealisten) stellt nun der sogenannte Euthypron-Kontrast dar: 
„If we suppose that all and only pious acts are loved by the gods, the biconditional

For any act $\mathrm{x}: \mathrm{x}$ is pious if and only if it is loved by the gods

is common ground between Socrates and Euthyphro. And a natural enough way of gesturing at the difference between them is to say that Socrates accords a certain priority to the left-hand side of the biconditional, while Euthyphro accords priority to its right-hand side. Style the first view detectivism about piety; and the second, projectivism." (Wright (1992): 108)

Bei der Position, die annimmt, daß die Götter die Frömmigkeit bestimmter Handlungen in der Welt erkennen oder detektieren, handelt es sich um einen Objektivismus oder lokalen Realismus hinsichtlich der Frömmigkeit: die Götter mögen bestimmte Handlungen besonders, gerade weil sie fromme Handlungen sind. Umgekehrt geht der Subjektivist oder Antirealist von einer Projektion der Eigenschaft der Frömmigkeit auf in der existierende Entitäten aus: bestimmte Handlungen gelten genau deswegen als fromm, weil sie von den Göttern bevorzugt werden. Der Unterschied besteht also darin, wie das betreffende Bikonditional gedeutet wird: welche ontologische Bestimmungs- oder Begründungsrichtung (order of determination) durch die Beschaffenheit der Realität vorgegeben ist. Entweder gibt es eine götter-unabhängige Frömmigkeit in der Welt, die von den Göttern jedoch als solche erkannt werden kann; oder aber die Frömmigkeit ist responsedependent, das heißt, sie wird erst durch die Reaktionen der Götter bestimmten Handlungen auferlegt.

Der Euthypron-Kontrast kann nun auch auf die Farbdebatte übertragen werden, indem das Bikonditional (BK) entsprechend ausgedeutet wird. Demnach geht der Objektivismus davon aus, daß Farbtatsachen unabhängig von den Reaktionen der farbwahrnehmenden Subjekte - also unabhängig von den bewirkten Farbwahrnehmungen - bestehen, während der Subjektivist gerade diese ontologische Abhängigkeit annimmt. Entweder erscheinen uns Gegenstände unter Normalbedingungen als rot, weil sie tatsächlich rot sind; oder sie gelten nur deshalb als rot, weil sie uns so normalerweise erscheinen. Führt die Analyse der Farben zu einer Identifikation 
mit den Eigenschaften $P$, dann können diese sowohl eine ontologisch subjekt-unabhängige (etwa eine rein physikalische) als auch eine ontologisch subjekt-abhängige (etwa eine dispositionale) Natur aufweisen. Die Differenz zwischen detektivistischer und projektivistischer Lesart kann auch hinsichtlich der Referentialität von Farbausdrücken formuliert werden. Während der Objektivist davon ausgeht, daß Farbausdrücke rigide referrieren, behauptet der Subjektivist eine attributive Referenz für sie. Das folgende Weltenbeispiel soll dies erläutern helfen.

Gehen wir von einem gegebenen Gegenstand aus, der eine bestimmte physikalische Beschaffenheit besitzt, aufgrund derer er in der aktualen Welt einem normalsichtigen Menschen unter typischen Wahrnehmungsbedingungen immer als rot erscheint. Wird der Gegenstand nun - ohne selbst in seiner Beschaffenheit verändert zu werden - in eine andere, nahe mögliche Welt gebracht und von einem dort befindlichen, menschlichen Betrachter wahrgenommen, so kann es der Fall sein, daß er für diesen nicht rot, sondern grün aussieht: etwa weil sich das visuelle System des Menschen oder die Bedingungen in der Welt verändert haben. Die Frage ist nun, ob der Gegenstand trotz des Transportes seine Farbe behalten oder ob er sie aufgrunddessen gewechselt hat. Oder auf sprachlicher Ebene formuliert: ob es also korrekt ist, den Gegenstand (im Vergleich zum aktualen Gebrauch von Farbausdrücken) auch in der möglichen Welt immer noch mittels des Wortes „Rot" zu charakterisieren, oder ob er doch besser „Grün“ genannt werden sollte (für einen Repräsentationalisten mag sich diese Frage nicht wirklich stellen, da er solche Inversionsbeispiele ablehnt). Werden Farbtatsachen nun als subjekt-unabhängig aufgefaßt, so gibt die aktuale Verwendungsweise auch die Referenz in anderen möglichen Welten vor: Farbausdrücke referrieren somit rigide. Dagegen faßt der Subjektivist Begriffe wie „Rot" und „Grün“ als attributiv auf: sie beziehen sich immer auf diejenigen Eigenschaften, die in der betreffenden Welt die entsprechenden Farbwahrnehmungen hervorrufen.

Da jedoch die linguistische Unterscheidung letztlich nur durch den Bezug auf die ontologische aufrechterhalten und erklärt werden kann, reicht es aus, sich hier auf letztere zu konzentrieren. ${ }^{1}$ Für den Objektivisten behält

1 Es gibt Versuche, die Farbproblematik auf dem umgekehrten Weg zu lösen (vgl. die Debatte zwischen Nida-Rümelin (1997) und Spohn (1997)), aber auch diese Versuche müssen sich schließlich - mehr oder weniger explizit - wesentlich auf 
der Gegenstand auch nach dem Transport seine Farbigkeit, weil sich seine ontologisch subjekt-unabhängige Beschaffenheit nicht geändert hat und die Tatsache, wie er einem Subjekt unter Normalbedingungen erscheint, nur kontingent bestehen muß. Rigidität entspricht also der Intuition, daß die Farbigkeit von Gegenständen auf deren intrinsischen Eigenschaften - und nur diesen - superveniert. Demgegenüber ändert sich die Farbe des Gegenstandes nach der subjektivistischen Ansicht, da für diese die Farbwahrnehmungen innerhalb der Subjekte den ontologisch relevanten Maßstab darstellen. Attributivität ist also ein Indiz dafür, daß Farbtatsachen auf dem Umstand supervenieren, wie sie einem Betrachter unter Normalbedingungen in der jeweiligen Welt erscheinen. Das Weltenbeispiel selbst wirft noch einige Fragen auf, die jedoch erst im weiteren Verlauf der allgemeinen Diskussion der Entgegensetzung von lokalem Farbrealismus und -antirealismus wieder aufgegriffen werden sollen. Doch die, wie gerade gesehen, für den Objektivismus so bedeutsame Rigidizitätsintuition läßt sich schon jetzt formulieren: wenn ein Gegenstand seine intrinsische Beschaffenheit nicht verändert, dann kann er auch nicht seine Farbigkeit verändern. Der Objektivismus sollte dieser Intuition möglichst gerecht werden.

\subsubsection{Metaphysische Notwendigkeit als Kriterium}

Zwei wichtige Punkte müssen nun geklärt werden: erstens muß ein handfesteres und anwendbareres Kriterium als die ontologische Begründungsrichtung für die Differenzierung von Subjektivismus und Objektivismus gefunden werden; und zweitens ist eine genauere Spezifierung der relevanten Subjektreaktionen erforderlich, damit die Grenze zwischen den beiden Alternativen klar gezogen werden kann. Beginnen wir mit der Fragestellung, welche Reaktionen in Subjekten für den Euthypron-Kontrast, das heißt, für die Formulierung des Bikonditionals, in Frage kommen. Da die Möglichkeit einer Geistabhängigkeit geben sein muß (ansonsten würde es sich in jedem Fall um einen Objektivismus handeln), sollten die fraglichen Reaktionen mentale Zustände der einen oder anderen Art sein. Im allgemeinen fordert Wright für einen bestimmten Diskurs, hinsichtlich dessen die Frage ontologische Überlegungen und Argumenationen stützen. 
nach dem ontologischen Status der betreffenden Tatsachen gestellt werden soll, allein das Vorhandensein beurteilender, das heißt, begrifflich ausgeformter Reaktionen. Bei vielen Diskursen muß man sich auch mit solchen Urteilen begnügen, da wahrscheinlich gar keine nichtbegrifflichen, phänomenalen oder auf irgendeine Weise sinnlichen Reaktionen möglich sind. Für Wright betrifft dies nicht nur den Bereich der Mathematik und der Bewertung von Modalitäten, sondern insbesondere auch den ethischen Diskurs: in allen drei Fällen gibt es keine Möglichkeit, durch eine wie auch immer beschaffene Art der Erfahrung Zugriff auf die entsprechenden Tatsachen zu erhalten (vgl. Wright (1992): 111; Wright (1988): 11ff.; 25f.).

Die Debatte über die sekundären Qualitäten bezieht hingegen natürlich in erster Linie die affektiven, sinnlichen Reaktionen - die inneren oder äußeren Sinneswahrnehmungen - ein, wie auch die bisherigen Formulierungen der Bikonditionale gezeigt haben. Die Farbwahrnehmungen sind die mentalen Zustände, die die unmittelbarste Reaktion auf (objektive oder subjektive) Farbtatsachen in der Welt darstellen. Farburteile sind dagegen immer epistemisch abhängig von Sinneserfahrungen (von vergangenen oder gegenwärtigen, von eigenen oder von solchen, die andere Subjekte haben oder gehabt haben und uns bereits durch ein ausgedrücktes Urteil mitteilen). Vor diesem Hintergrund kann jetzt die Frage zu beantworten versucht werden, welches Kriterium sinnvollerweise helfen kann, den Unterschied zwischen den beiden ontologischen Lesarten des Bikonditionals deutlich herauszuheben, so daß eine abwägende Diskussion beider Alternativen möglich wird. Einen wesentlichen Beitrag hierzu hat Crispin Wright geleistet, dessen Argumentation hier in Teilen rekonstruiert werden soll. ${ }^{2}$ Die Abfolge und Formulierung seiner Überlegungen wird dabei an den hier gewählten Farbdiskurs angepaßt. Zur Erinnerung soll deshalb nochmals das - „uninterpretierte“ - Bikonditional für Farben angegeben werden (wobei das $F_{\mathrm{i}}$-Quale, wie erläutert, paradigmatisch in der aktualen Welt festgelegt wird):

2 Die folgenden Ausführungen beziehen sich hauptsächlich auf den Appendix The Euthyphro Contrast: Order of Determination and Response-Dependence in seinem Buch Truth and Objectivity (Wright (1992)). Weiterhin zu beachten sind Wright (1988), (1998) und Johnston (1993). Zu Wrights genereller, anti-realistischer Grundhaltung: vgl. weiter unten, Kap. 5, Fn. 27. 
(BK) Farbe $F_{\mathrm{i}} \equiv P_{\mathrm{i}} \leftrightarrow$ [in der Welt $\mathrm{w}$ gilt: $P_{\mathrm{i}}=$ unter Normalbedingungen mit dem $F_{\mathrm{i}}$-phänomenalen Wahrnehmungstypus $W_{\mathrm{i}}$ von Subjekten $S$ nomologisch korrelierte Eigenschaft].

Dieses Bikonditional entspricht in seiner logischen Form dem ganz zu Anfang im Zusammenhang mit der Analysethese (ANA) vorgestellten Bikonditional: (Farben $F \equiv$ Eigenschaften $P$ ) $\leftrightarrow$ (die Eigenschaften $P$ weisen $\Phi$ auf). Das Merkmal $\Phi$ ist dabei lediglich mit der Eigenschaft gleichgesetzt worden, unter Normalbedingungen mit einen bestimmten phänomenalen Wahrnehmungstyp nomologisch korreliert zu sein. Bei dieser Eigenschaft handelt es sich um ein Merkmal, daß den Farben zumindest faktisch zukommt, so daß sie in der aktualen Welt hierüber als Farben identifiziert werden können. Die spätere Strategie für die einzelnen Theorien der Farben wird ganz einfach sein: finde die Eigenschaften $P$, die dieses Merkmal $\Phi$ aufweisen; dann hast du den besten Kandidation für die Identifikation mit den Farben. Das hier in (BK) verwendete Merkmal $\Phi$ wird dabei den Farben mindestens kontingent in der aktualen Welt zukommen (dies drückt das normale Gleichheitszeichen „=“ aus). Die linke Seite des Bikonditionals ist somit wenigstens aktual wahr. Doch eine Theorie der Farben kann zudem fordern, daß diese das Merkmal notwendigerweise in allen möglichen Welten zeigen. Und schließlich kann es sich auch noch um eine begriffliche Wahrheit handeln. Die linke Seite von (BK) kann also drei verschiedene Modalitätsstufen annehmen. Da die rechte Seite zumindest eine notwendige Identität a posteriori ausdrückt, stehen hier nur zwei Alternativen zur Auswahl: Aposteriorität (welche dem Zeichen „„“ entspricht) oder Apriorizität (die durch ,:=“ bezeichnet wird). Wie es sich herausstellen wird, gibt es sinnvollerweise damit die drei folgenden, möglichen Interpretationen des Bikonditionals (welches auf jeden Fall im ganzen metaphysische Notwendigkeit aufweisen wird):

\begin{tabular}{|c|c|c|c|}
\hline (BK) & linke Seite & rechte Seite & Modalität \\
\hline Objektivismus $_{\mathrm{ACT}}$ & $F \equiv P$ & $P=\Phi$ & a posteriori \\
\hline
\end{tabular}




\begin{tabular}{|c|c|c|c|}
\hline Objektivismus $_{\mathrm{NEC}}$ & $F \equiv P$ & $P \equiv \Phi$ & a posteriori \\
\hline Subjektivismus & $F:=P$ & $P:=\Phi$ & a priori \\
\hline
\end{tabular}

Im folgenden soll diese Zuordnung der drei Positionen hergeleitet werden. Trifft sie zu, dann entspricht dem Unterschied zwischen Objektivismus und Subjektivismus der Gegensatz von Aposteriorität und Apriorizität hinsichtlich des Bikonditionals (und hinsichtlich der beiden Seiten für sich genommen). Ob metaphysische Kontingenz oder Notwendigkeit in der rechten Seite von (BK) vorliegt, kann zudem zu einer Unterteilung der objektivistischen Position führen. Da diese Differenz nur in der rechten Seite aufzufinden sein wird, lohnt es sich, sich in der weiteren Diskussion besonders auf diese zu konzentrieren. Theoretisch kann man deshalb statt dem ganzen Bikonditional auch nur die rechte Seite betrachten, indem man die Eigenschaften $P$ als „Vermittler“ zwischen den Farben und dem Merkmal $\Phi$ ganz herausnimmt und durch die Farben selbst ersetzt. Somit ergäbe sich etwa die folgende, zu überprüfende Aussage: $F=\Phi$. Doch die begriffliche Trennung von den $F$ - und den $P$-Eigenschaften ist bereits im Kontext der Analysethese begründet worden: erst sie macht wirklich deutlich, daß es darum geht, daß eine Intuitive Farbkonzeption theoretisiert werden soll. Die Aussage $F=\Phi$ ist als Desiderata bereits Bestandteil der Intuitiven Farbkonzeption. Damit also eine erfolgreiche Analyse der Natur der Farben vorliegen kann, müssen die Analysanda $P$ ebenfalls dieses Merkmal zeigen. Aus dieser Überlegung heraus ist das Bikonditional (BK) der einfachen Formulierung vorgezogen worden.

Der Objektivist wird nun die Identifikation auf der linken Seite als maßgebend ansehen: für ihn wird die Natur der Farben allein mithilfe der $P_{\mathrm{i}}$ angemessen analysiert, wobei letztere ganz unabhängig von den Farbwahrnehmungen der Subjekte $S$ spezifiziert und untersucht werden können. Es ist also von vorneherein nicht zwingend, daß $F_{\mathrm{i}}$-Farbtatsachen unter wirklich allen möglichen Umständen zu Farbwahrnehmungen mit einem $F_{\mathrm{i} \text { - }}$ Quale führen müssen. Deshalb genügt es dem Objektivisten, für den rechten Teil von $(\mathrm{BK})$ nur metaphysische Kontingenz zu fordern: nur für die aktuale Welt muß die These wahr sein, daß die $P_{\text {i }}$ entsprechende $F_{\mathrm{i}}$-Wahrnehmungen in Menschen bedingen, aber in anderen möglichen Welten - 
mit anderen farbwahrnehmenden Subjekten oder anderen Naturgesetzmäßigkeiten - muß dies nicht unbedingt der Fall sein (wenigstens nicht ohne die Berücksichtigung zusätzlicher Annahmen oder Überlegungen). Dagegen wird der Subjektivist Farben ontologisch über die hervorgerufenen Farbwahrnehmungen analysieren: $F_{\mathrm{i}}$ ist demnach nichts anderes als die Ei-

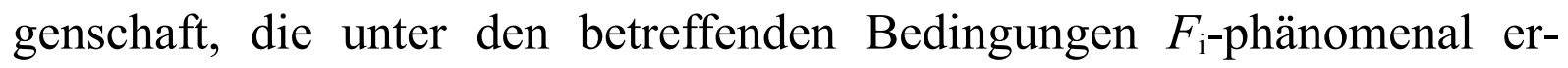
scheint. Der rechte Teil von (BK) muß demnach eine metaphysisch notwendige Identität ausdrücken: er gilt in allen Welten w und für alle farbwahrnehmende Subjekte darin. ${ }^{3}$

Vor diesem Hintergrund kann Wright nun als ein erstes, vorläufiges Kriterium einen metaphysischen Modalitätsunterschied ausmachen. Da auch die linke Seite von (BK) eine metaphysisch notwendige Aussage trifft, muß für den Subjektivisten das gesamte Bikonditional metaphysisch notwendig wahr sein. Der Objektivist muß im Gegensatz dazu nur metaphysische Kontingenz fordern: auch wenn der linke Teil des Bikonditionals in allen Welten wahr ist, muß dies nicht auch unbedingt für den rechten gelten. Aufgrund dieses Unterschiedes ergibt sich jedoch eine erste Konsequenz: und zwar dürfen die Normalbedingungen nicht trivial sein, sondern müssen substantiell bestimmt werden. Triviale Normalbedingungen lassen sich in etwa wie folgt definieren: genau die Bedingungen, die notwendig sind, damit die Eigenschaften $P_{\mathrm{i}}$ in Subjekten $S$ in der betreffenden Welt w Farb wahrnehmungen des $F_{\mathrm{i}}$-phänomenalen Types hervorrufen. Doch unter dieser Voraussetzung nimmt die rechte Seite von (BK) sowohl für den Objektivisten als auch für den Subjektivisten nicht nur die gleiche Form an, sie wird auch für beide trivialerweise in allen Welten wahr - nur mit dem Unterschied, daß nach der objektivistischen Auffassung in vielen Welten die so definierten Normalbedingungen faktisch leer bleiben, während der Subjektivist für alle Welten Normalbedingungen angeben können wird.

Damit dieser Unterschied sich aber, wie angestrebt, bereits in der Formulierung von $(\mathrm{BK})$ zeigt, müssen die Normalbedingungen anhand empirischer Forschungen und statistischer Berechnungen näher bestimmt werden. ${ }^{4}$ Somit erhält man eine substantielle, statistische Charakterisierung der

3 Wright selbst beschränkt sich zwar im Grunde auf eine einzige Subjektgruppe, die Menschen, aber er spricht sowohl die Möglichkeit anderer Subjekte als auch anderer Wahrnehmungsbedingungen an (vgl. Wright (1992): 113).

4 Vgl. den Abschnitt über Normalbedingungen. Als Beispiele für eine solche sub- 
Normalbedingungen (vgl. Wright (1992): 112). Hiermit gibt es jedoch erneut ein Problem. Die Normalbedingungen, wie sie aktual für menschliche Betrachter bestehen, können sich natürlich ändern: die physiologische Beschaffenheit von Menschen könnte sich ändern, aber auch die äußeren Wahrnehmungsbedingungen. Es muß also noch geklärt werden, welche Normalbedingungen einer statistischen Untersuchung unterzogen werden sollten. Hierbei gibt es grundsätzlich zwei Möglichkeiten: entweder werden für jede betrachtete Welt immer nur die Normalbedingungen der aktualen Welt als Maßstab genommen; oder es werden die jeweiligen, in der möglichen Welt w vorherrschenden Normalbedingungen für Farbwahrnehmungen berücksichtigt. Es geht also darum, ob die Erwähnung der Normalbedingungen in (BK) sich immer auf die aktualen oder aber auf die faktischen, das heißt, auf die in der betreffenden möglichen Welt w bestehenden Normalbedingungen bezieht.

Die letztere Alternative hat jedoch die ungewollte Konsequenz, daß sie die Differenz zwischen Subjektivismus und Objektivismus davon abhängig macht, was in möglichen Welten über die Korrelationen von wahrgenommenen Tatsachen und Farbwahrnehmungen gilt. Oben ist bereits, im $\mathrm{Zu}$ sammenhang mit dem Weltenbeispiel, der Unterschied zwischen rigider und attributiver Referenz von Farbausdrücken in normalen Farburteilen (wie „dieser Gegenstand ist rot") eingeführt worden, wobei erstere Lesart dem Objektivismus, und letztere dem Subjektivismus entspricht. Nun darf eigentlich der Umstand, daß im allgemeinen substantielle, für die jeweilige Welt spezifische Normalbedingungen angenommen werden, für sich genommen noch keine Entscheidung zwischen den beiden Positionen herbeiführen. Dies sollte erst durch die konkrete Bestimmung der Normalbedingungen erreicht werden. Das heißt, der Beschluß, die Normalbedingungen generell relativ zu den jeweiligen betrachteten Welten zu bestimmen, darf noch keine Vorentscheidung für eine der beiden ontologischen Auffassungen darstellen. Insbesondere sollte die folgende

stantielle Angabe der Normalbedingungen können zum Beispiel die CIE-Standards für Betrachter und Wahrnehmungsbedingungen angeführt werden (vgl. Hardin (1988): 209f.). Eine andere, ,philosophischere“ Ausformulierung hinsichtlich von Rot- und Quadratischwahrnehmungen sowie moralischen Urteile findet sich in Wright (1988). 
Rigiditätsintuition des Objektivisten zumindest noch möglich bleiben (auch dann, wenn eine konkrete Ausformulierung der jeweiligen Normalbedingungen ergeben wird, daß die subjektivistische, attributive Lesart letztlich angebrachter ist).

„We are after a sharp, operationally applicable account of the - or at least a - Euthyphro distinction. But we cannot ab initio foreclose on the possibility that, when we have gained it, colours and shapes may fall out on the same side. [...]

We are therefore obliged to pay attention to the following intuition about colour: that had the typical visual equipment of human beings been very different, or had the lighting (by day) on the earth typically been of a quite different character perhaps resembling the illumination generated by sodium street lighting - that need have made no difference to the colours things actually are. The extensions of ,red“ and „green“ would not have been different if all human beings had been colour blind, and would not change if they were to become so.

The intuition is, effectively, that the colour of an object supervenes on its intrinsic physical character.“ (Wright (1992): 113)

Man kann sich nun aber überlegen, wann es für einen Objektivisten möglich ist, diese Rigiditätsintuition aufrechtzuerhalten. Dabei spielt die Frage, ob Veränderungen in den wahrnehmenden Subjekten, den Wahrnehmungsbedingungen oder auch den zugrundeliegenden Naturgesetzmäßigkeiten auch automatisch zu Veränderungen in der Phänomenalität der Wahrnehmungen führen, und wenn ja, ob dann erstere Veränderungen überhaupt möglich sind, eine entscheidende Rolle. Eine Antwort darauf wird meistens im Zusammenhang mit den einzelnen Varianten von Qualia-Inversion (und auch Qualia-Abwesenheit) disktutiert, aber es gibt auch andere Beispiele, die die eine oder die andere Alternative plausibler machen sollen (vgl. die Abschnitte über Qualia und über den Objektivismus ${ }_{\mathrm{NEC}}$ ). Betrachten wir zunächst Positionen, die davon ausgehen, daß Qualia-Inversion unmöglich ist (diese Fälle werden von Wright nicht diskutiert, da er ohne weitere Erläuterung die Möglichkeit von solchen Inversionen voraussetzt (vgl. Wright (1992): 114)). Wird ein Gegenstand also aufgrund einer bestimmten intrinsischen Eigenschaft als farbig repräsentiert, so wird dieser Gegenstand in jeder möglichen Welt mit ein- und derselben Farbphänomenalität gesehen, solange immer dieselbe, unveränderte intrinsische Eigenschaft 
des Objektes repräsentiert wird. ${ }^{5}$

Hierbei handelt es sich aber um nichts anderes als die Notwendigkeitsthese, derzufolge Gehalt und Quale in allen möglichen Welten so zusammenauftreten, wie sie es in der aktualen Welt tun. Es ist somit zum Beispiel notwendig, daß ein roter Gegenstand in allen Welten, in denen er überhaupt Farbwahrnehmungen verursachen kann, unter den jeweiligen Normalbedingungen Rotwahrnehmungen bewirkt. Dabei ist die These erst einmal davon unabhängig, ob die Normalbedingungen in verschiedenen Welten unterschiedlich sind oder nicht: sie schließt diese Möglichkeit jedenfalls nicht von vorneherein aus (dieser Punkt wird während der Argumentation

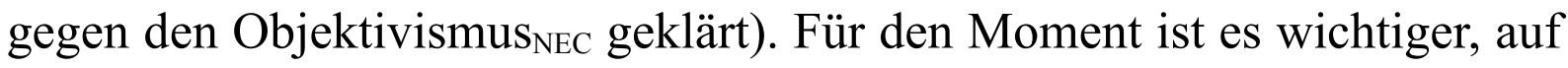
den Umstand der Notwendigkeit hinzuweisen. Denn die Akzeptanz der Notwendigkeitsthese $\left(\mathrm{NEC}_{\mathrm{NB}}\right)$ bringt es automatisch mit sich, daß die rechte Seite des Bikonditionals (BK) notwendig wahr wird, ganz gleich, ob die Normalbedingungen in verschiedenen Welten differieren oder nicht. Dies hat jedoch zur Konsequenz, daß die Anwendung des metaphysischen Notwendigkeitskriteriums dazu führen würde, daß jede Position, die Qualia-Inversion ablehnen und $\left(\mathrm{NEC}_{\mathrm{NB}}\right)$ akzeptieren, ein Subjektivismus wäre.

Indessen impliziert die Akzeptanz von Qualia-Inversion und die Ablehnung der Notwendigkeitsthese, daß die rechte Seite des Bikonditionals nur in den Welten wahr ist, in denen ein intrinsisch unveränderter Gegenstand zu Farbwahrnehmungen führt, die denen, die er in der aktualen Welt hervorruft, phänomenal gleichen. Da es wegen der Inversionsmöglichkeit viele mögliche Welten geben wird, die dies nicht erfüllen, kann (BK) nur kontingent wahr sein: es würde sich also, nimmt man erneut metaphysische Notwendigkeit als Kriterium, um einen Objektivismus handeln. Das heißt aber, daß, sobald man die Normalbedingungen in (BK) welt-relativ bestimmt, die Unterscheidung zwischen Objektivismus und Subjektivismus sich allein auf die ontologische Möglichkeit oder Unmöglichkeit von Qua-

5 Die Repräsentationalisten, die diese These vertreten, wenden meistens eine der beiden folgenden Strategien an, um Inversionsbeispiele zu widerlegen. Erstens können die Veränderungen der Normalbedingungen so gedeutet werden, daß sie zu einem Wechsel der repräsentierten Eigenschaft führen (oder zu einem Verlust der Repräsentationalität), so daß der phänomenale Unterschied sich wiederum auf den Gehaltsunterschied zurückführen läßt. Zweitens können die Veränderungen aber auch als metaphysisch oder logisch unmöglich dargestellt werden (vgl. Tye (1992); (1995): Kap. 7). 
lia-Inversion reduzieren ließe. Das kann aber nicht so ohne weiteres der Fall sein. Denn es wird nicht nur in einem späteren Kapitel sogar dafür argumentiert werden, daß der Repräsentationalismus, welcher $\left(\mathrm{NEC}_{\mathrm{NB}}\right)$ akzeptiert, sogar zu einem Farbobjektivismus gezwungen ist (vgl. den Abschnitt über den Objektivismus $\mathrm{NEC}_{\mathrm{NE}}$ ); sondern auch Überlegungen anhand des Weltenbeispieles zeigen, daß die Unmöglichkeit von Qualia-Inversion sowohl subjektivistisch als auch objektivistisch gedeutet werden kann.

Betrachten wir dazu wieder den Gegenstand, der ohne eine Veränderung seiner physikalischen Beschaffenheit in eine andere mögliche Welt transportiert wird. Aus der Notwendigkeitsthese, welche die Ablehnung von Qualia-Inversion formuliert, ergibt sich aber, daß der Gegenstand in allen Welten unter welt-relativ spezifzierten Normalbedingungen gleichfarbig aussehen wird. Nun macht es zwar immer noch Sinn, den Euthypron-Kontrast angesichts der Farben aufrechtzuerhalten und zu fragen, ob Farbausdrücke rigid oder attributiv referrieren: ob also der Gegenstand in der neuen, möglichen Welt seine rote Farbe hat, weil er dieselbe intrinsische Beschaffenheit wie in der aktualen aufweist, oder weil er in der fragliche Welt genauso wie in der aktualen aussieht. Aber eine Antwort läßt sich anhand des Einbeziehens von möglichen Welten gar nicht mehr geben, da sich diese hinsichtlich der Korrelation von Farbtatsachen und hervorgerufenen Farbwahrnehmungen nicht von der aktualen unterscheiden. Die Betrachtung der durch die Notwendigkeitsthese bedingten metaphysischen Notwendigkeit von (BK) liefert also ein voreiliges Urteil ab: auch wenn in diesem Fall das Bikonditional notwendig wahr ist, ist es doch mit einem Objektivismus, das heißt, mit der Rigiditätsintuition kompatibel.

Geht man hingegen von der Möglichkeit von Qualia-Inversionen aus, dann kann (BK) gar nicht mehr metaphysisch notwendig werden, wenn man welt-spezifische Normalbedingungen hat, da es immer Welten geben wird, in denen Gegenstände anders aussehen werden als in der aktualen Welt, obwohl sie intrinsisch unverändert bleiben. Dies ist ein Sonderfall der These, daß es für einen Subjektivisten überhaupt keinen Sinn macht, von einem Auseinandergehen von Gehalt und Quale zu sprechen. Da der Umstand, welche Farbeigenschaft gerade repräsentiert wird, immer von dem phänomenalen Aspekt der Farbwahrnehmungen bestimmt wird (und nicht umgekehrt), kann es gar nicht passieren, daß repräsentierte Farben und Farbqualia in einer anderen Welt auf verschiedene Weise miteinander 
korreliert sind (natürlich können die intrinsischen, physikalischen Realisatoren der Farben jeweils sehr verschieden sein: sowohl innerhalb der aktualen als auch zwischen möglichen Welten im Vergleich). Somit trifft das Kriterium der metaphysischen Modalität wenigstens die halbe Wahrheit, indem es die Kontingenz von (BK) allein für den Objektivismus reserviert. ${ }^{6}$ Doch für einen Subjektivismus erweist sich das Kriterium, für sich genommen, als inadäquat.

Hilft es dann vielleicht weiter, die Normalbedingungen nicht welt-relativ zu bestimmen, sondern immer die aktual vorherrschenden als Maßstab zu nehmen? Es ist klar, daß die Konzentration auf die aktuale Welt einige Vorteile bietet. Zum einen unterliegt die Diskussion nicht mehr der Ungenauigkeit von Spekulationen, sondern kann sich voll und ganz auf empirisch gewonnene Erkenntnisse über das Farbensehen stützen. Zum anderen ist damit die Frage nach der rigiden oder attributiven Verwendungsweise von Farbausdrücken und die entsprechende ontologische Differenz ganz von der Frage nach der Möglichkeit oder Unmöglichkeit von QualiaInversionen getrennt. Wright diskutiert diese Alternative der Aktualisierung

$6 \quad$ Wright sieht auch hierfür ein Problem, da er die Rigiditätsintuition unter der Voraussetzung von welt-relativen Normalbedingungen sowie der Möglichkeit von Qualia-Inversion generell verletzt sieht:

„For there seems to be no difficulty in conceiving, for instance, of alterations of the laws of nature of such a kind that objects whose surfaces we perceive as coloured thus-and-such would, without undergoing any intrinsic alteration, induce dramatically different visual effects in us, and would appear as they now appear to us only to creatures with a radically different visual physiology. Insisting, in the teeth of this possibility, that basic equations for colours, formulated as proposed [i.e., (BK) - der Autor], were necessarily true would amount, once again, to repudiating the above intuition - to insisting that intrinsically unaltered objects would, in certain possible circumstances, rightly be regarded as having changed in colour." (vgl. Wright (1992): 114)

Es ist zwar richtig, daß eine Position, die Farben über Farbwahrnehmungen analysiert, unter der Annahme von Qualia-Inversion der Rigidizitätsintuition nicht gerecht werden kann. Aber bei solch einer Position wird es sich um einen Subjektivismus handeln, der in jedem Fall diese Intuition zurückweisen wird. Der Objektivismus kann sich der Schlußfolgerung Wrights entziehen. 
(oder „Rigidifizierung“, wie er es nennt) von Normalbedingungen und kommt zu dem Schluß, daß diese das bisherige Kriterium für die Differenz zwischen Subjektivismus und Objektivismus aller Voraussicht nach ebenso nicht - oder nur unter sehr großen Anstrengungen - aufrechterhalten kann.

Die allgemeine Argumentation hierfür ist recht einfach (vgl. Wright (1992): 114ff.). Die rechte Seite des Bikonditionals nimmt nach der Aktualisierung in etwa die folgende Form an: in der aktualen Welt, gilt, daß die mit der Farbe $F_{\text {i }}$ gleichzusetzende Eigenschaft $P_{\text {i }}$ unter (aktualen) Normalbedingungen in (aktualen) Subjekten immer zu $F_{\mathrm{i}}$-phänomenalen Farb wahrnehmungen führt. Hierfür gibt es jedoch ein Problem, wenn es sich tatsächlich herausstellen sollte, daß die so formulierte These innerhalb des Aktualitätsskopus der rechten Seite von (BK) faktisch wahr ist: wovon aufgrund der generellen Akzeptanz der Aktualitätsthese $\left(\mathrm{ACT}_{\mathrm{NB}}\right)$ durch sowohl objektivistische als auch subjektivistische Farbtheorien ausgegangen werden kann. Denn ist eine Aussage $p$ für die aktuale Welt wahr, so wird eine Aussage $q$, die den Aktualitätsoperator auf $p$ anwendet - also die These, daß $p$ aktual wahr ist -, in allen Welten Gültigkeit haben und somit notwendig wahr sein. Jede aktuale Wahrheit ist notwendigerweise aktual wahr. Die Aktualisierung bringt also mit sich, daß das Bikonditional auf jeden Fall metaphysisch notwendig wahr wird: und somit kann die metaphysische Modalität wieder nicht als Kriterium für den Euthypron-Kontrast dienen. Wright diskutiert nun eine komplizierte Möglichkeit, dieses Problem vielleicht durch eine Modifikation des Skopus des Aktualitätsoperators lösen zu können.

„We might seek a more fine-grained way of rigidifying a broadly statistical account of the appropriate conditions of observation without thereby rigidifying the physical laws which actually sustain or are somehow implicated in those conditions. And in that case counterexamples might yet be forthcoming...: worlds in which, owing to operation of different physical laws, observers having actually typical visual equipment would not, under conditions of observation like those which actually typically obtain, in general receive faithful impressions of shape.“ (Wright (1992): 116)

Wie hier für Formen besteht also immer noch eine Hoffnung auf die Möglichkeit, daß das Bikonditional trotz der Aktualisierung (oder Rigidifizierung) kontingent bleiben könnte. Um diese Möglichkeit zufriedenstellend 
zu testen, wird man jedoch etlichen Schwierigkeiten hinsichtlich der - auf eine bestimmte mögliche Welt bezogegen - Trennung von Tatsachen in solche, die von den Naturgesetzmäßigkeiten herrühren, und in solche, die von diesen gänzlich unbeeinflußt bleiben, auf die eine oder andere Weise aus dem Weg gehen müssen: ohne wirklich zu wissen, ob zuguterletzt die Hoffnung sich auch tatsächlich bestätigen wird. Wright entzieht sich diesen weiteren Komplikationen, indem er auf metaphysische Modalität als Kriterium für die Unterscheidung von Subjektivismus und Objektivismus hinsichtlich von Farben (oder anderen Eigenschaften) ganz verzichtet (vgl. Wright (1992): 116). Die allgemeine Plausibilität einer solchen Wahl der Alternative mit dem geringsten Widerstand läßt sich in diesem Fall zudem noch konkret begründen. Denn es ist bereits auf das wesentliche Problem hingewiesen worden, daß im Falle der Akzeptanz der Notwendigkeitsthese $\left(\mathrm{NEC}_{\mathrm{NB}}\right)$ auch die Aktualisierung nicht wird verhindern können, daß (BK) auf alle Fälle metaphysisch notwendig wird.

\subsubsection{Epistemische Notwendigkeit als Kriterium}

Die Aufgabe der metaphysischen Notwendigkeit als Kriterium für den Subjektivismus ist bei Wright mit einer Hinwendung zur epistemischen Notwendigkeit verbunden (vgl. Wright (1992): 116f.). Es ist bereits gezeigt worden, daß die Kontingenz des Bikonditionals (BK) immer auf einen Objektivismus hinweist. Zudem ist argumentiert worden, daß metaphysische Notwendigkeit allein noch nicht ausreicht, um subjektivistische Positionen von objektivistischen zu unterscheiden. Denn diese Notwendigkeit kann sich nur aus der Aktualisierung oder als eine direkte Folge der zusätzlich angenommenen Notwendigkeitsthese $\left(\mathrm{NEC}_{\mathrm{NB}}\right)$ ergeben: was beidesmal mit einem Objektivismus verträglich ist. Doch weder die Aktualisierung, noch die Notwendigkeitsthese reicht aus für eine epistemische Notwendigkeit des Bikonditionals, da beide Überlegungen nur die ontologische, nicht jedoch die epistemische Ebene betreffen. Damit läßt sich die Debatte um die Begründungsrichtung beim Euthypron-Kontrast formulieren, indem epistemische Notwendigkeit oder Apriorizität gefordert wird. 
„I therefore imposed an Apriority condition: roughly, it will suffice to classify a class of judgements on the detectivist side of the Euthyphro contrast if, while they do sustain true basic equations [d.h. (BK) für einzelne Farben - der Autor], complying with the Substantiality condition, none of these basic equations can be known to be true a priori. Correspondingly, one might now suggest, it will be a necessary condition for the propriety of Euthyphronic viewpoint that appropriate, substantially specified basic equations can be known to be true a priori." (Wright (1992): 117)

Wright gibt also zu, daß eine vollständige Analyse des Euthypron-Kontrastes damit zwar noch nicht gegeben ist (es sind nicht für beide Positionen jeweils die hinreichende und die notwendigen Bedingungen bekannt), aber daß über die epistemische Modalität der Bikonditionale hinsichtlich eines bestimmten Eigenschaftsdiskurses (wie etwa des Farbdiskurses, der aus auf Wahrnehmungen beruhenden Urteilen wie „dies ist rot" besteht) die Objektivität oder die Subjektivität der betreffenden Tatsachen eindeutig festgestellt werden kann. Die Beantwortung der Frage, ob Farbtatsachen besser subjektivistisch oder objektivistisch analysiert werden, ist, gestützt durch empirische Erkenntnisse und philosophische Überlegungen, eines der Hauptziele dieser Diskussion, deren systematischer Grundaufbau ja hier geklärt werden soll. Die Meinung, daß der Subjektivismus nicht nur eine ontologische, sondern zudem eine logisch-begriffliche Abhängigkeit der Farbtatsachen von dem Auftreten der entsprechenden Farbwahrnehmungen in Subjekten beinhaltet, teilen neben Wright auch andere Philosophen mit, was Farben betrifft, zum Teil sehr unterschiedlichen Auffassungen. $^{7}$

„Secondary qualities are subjective in the sense that experience enters into their analysis: to grasp the concept red it is necessary to know what it is for something to look red, since this latter constitutes the satisfaction condition for an object's being red. [...] It is a conceptual truth that red things typically look red." (McGinn (1983): 8 ; 11)

„Rather than employing the subjective/objective distinction to distinguish physicalism from rival views, it is more useful to classify accounts of color as subjective to the extent that they claim a substantive a priori connection between being a

7 Auch Jackson nimmt für einen Subjektivisten eine essentielle Verbindung zwischen Farbtatsachen und Farbwahrnehmungen an (vgl. Jackson (1996): 208f.). 
certain color and causing certain visual experiences." (Einleitung zu Byrne \& Hilbert (1997a): xxiii)

„I, however, take Smart to be cutting all logical links between colours and what happens in the perceivers of colours. (It may be that I do this because I think that a complete objectivity, a complete realism, about the secondary qualities is the true view).“(Armstrong (1987): Fn. 13)

Es lassen sich nun hinsichtlich der rechten Seite von (BK) drei Modalitätsstufen unterscheiden: (i) Kontingenz; (ii) metaphysische Notwendigkeit; (iii) epistemische Notwendigkeit. Es ist dabei wichtig, herauszuheben, daß die Modalität der rechten Seite von (BK) automatisch auch die Modalität des gesamten Bikonditionals festlegt. Für die ersten beiden Modalitätsgrade ist dies sofort einsichtig, beachtet man, daß die linke Seite von (BK) auf alle Fälle in allen Welten gilt. Ein Bikonditional ist genau dann metaphysisch notwendig wahr, wenn die beiden Thesen, die es verbindet, (mindestens) diese Notwendigkeit zeigen; und es ist kontingenterweise wahr, wenn die beiden Thesen (mindestens) in der aktualen Welt zutreffend sind. In beiden Fällen ergibt sich der Grad der metaphysischen Modalität aus der Menge der möglichen Welten, in denen die betreffenden Aussagen wahr sind. Anders sieht es dagegen für die Forderung einer epistemischen Notwendigkeit für die rechte Seite von (BK) aus. Das Bikonditional besteht somit anscheinend aus zwei Thesen mit unterschiedlicher Modalitätsstärke, da die eine - die linke - nur metaphysische Notwendigkeit zeigt.

Dies täuscht jedoch, denn für einen Subjektivismus muß im Grunde diese linke Seite des Bikonditionale umformuliert werden. Bei der Analyse der Natur der Farben mittels der Eigenschaften P handelt es sich nicht wie noch im Falle des Objektivismus - um eine empirisch, sondern um eine begrifflich zu vollziehende Identifikation. Auf dieselbe Weise, wie die von uns „Wasser“" genannte Substanz durch eine chemikalische Analyse als Stoff mit der Molekülstruktur $\mathrm{H}_{2} \mathrm{O}$ identifiziert wird, wird der Objektivist aufgrund empirischer Forschungsergebnisse zu einer Gleichsetzung von Farben (also dem, was wir „Farben“ nennen) mit bestimmten, subjekt-unabhängig instantiierten Eigenschaften gelangen. In beiden Fällen ist jedoch ein Rückgriff auf die Ergebnisse der Empirie vonnöten, weswegen die Bestimmung der wesentlichen Charakteristika von Farben nur a posteriori sein kann: es liegt demnach nur metaphysische Notwendigkeit vor. 
Im Gegensatz dazu stipuliert der Subjektivismus (vor dem Hintergrund argumentativ oder empirisch gewonnener Einsichten, die gegen einen $\mathrm{Ob}-$ jektivismus zu sprechen scheinen) sozusagen die Natur der Farben, indem er die Gleichsetzung mit bestimmten, geistabhängigen Eigenschaften eben als eine begriffliche Identiät darstellend auffaßt. Die Natur dieser Eigenschaften wird vor aller Empirie festgelegt und kann gar nicht mittels einer Erforschung objektiver Tatsachen in der Welt analysiert werden (sieht man vielleicht von einer möglichen objektiven Beschreibung von Subjekten ab), weil sie eben eine ontologische Geistabhängigkeit aufweisen und in verschiedenen Welten anders physikalisch realisiert sein können. Die Identifikation der Farben $F$ mit den Eigenschaften $P$ hat damit bereits einen $a$ priori Charakter, so daß das Bikonditional insgesamt epistemische Notwendigkeit zeigt. ${ }^{8}$ Dies wird später auch eindeutige Konsequenzen für die konkrete Ausformulierung eines Farbsubjektivismus haben, der eine wesentliche, ontologische Abhängigkeit zwischen Farben und Farbwahrnehmungen annehmen muß.

Den drei Modalitätsstufen entsprechen nun drei ontologische Grundpositionen. Kontingenz weist auf einen Objektivismus hin, der die Notwendigkeitsthese $\left(\mathrm{NEC}_{\mathrm{NB}}\right)$ ablehnt, metaphysische Notwendigkeit hingegen auf einen Objektivismus, der diese These akzeptiert. Somit kann man von einem Kontingenz- und einem Notwendigkeitsobjektivismus sprechen (oder von einem Objektivismus ${ }_{\mathrm{ACT}}$ und einem Objektivismus NEC $_{\mathrm{NEC}}$ ). Demgegenüber ist ein Subjektivismus immer mit der epistemischen Notwendigkeit verbunden. In diesem Sinne kann ein Subjektivist sinnvollerweise keine Qualia-Inversion erlauben, wenn es um eine Korrelation zwischen den Farben und den Farbqualia geht. Dies ist aber auch nicht weiter schlimm, da normalerweise die Inversionen der Qualia hinsichtlich von objektiven, intrin-

8 Durch eine Umformulierung des Bikonditionals wird dies noch klarer, indem man einfach den Einschub der Analyseaussage $F_{\mathrm{i}} \equiv P_{\mathrm{i}}$ wegläßt.

$\left(\mathrm{BK}^{*}\right) \quad$ Farbe $F_{\mathrm{i}}=$ die in der Welt $\mathrm{w}$ unter Normalbedingungen mit dem $F_{\mathrm{i}^{-}}$ phänomenalen Wahrnehmungstypus $W_{\mathrm{i}}$ von Subjekten $S$ nomologisch korrelierte Eigenschaft.

Hierbei handelt es sich im Prinzip nur um die rechte Seite des ursprünglich betrachteten Bikonditionals: deren Modalitätsstufen gelten also auch für (BK*). 
sischen Eigenschaften der Objekte spezifiziert werden (wie etwa Reflektanzeigenschaften), und nicht hinsichtlich von geistabhängigen (wie den subjektiven Farben). Ein Subjektivismus kann also ohne weiteres über die Frage streiten, ob in allen Welten dieselben physikalischen Eigenschaften von Gegenständen mit einem ganz bestimmten Farbwahrnehmunsgtypus verbunden sind oder nicht. Für eine Theorie der Farben wird dies jedoch eine recht uninteressante Frage, da die Annahme einer multiplen Realisierbarkeit von Disposition - um die es hier alleine geht - nicht allzu sehr kontrovers ist. Für den Subjektivisten supervenieren Farbtatsachen eben nicht (nur) auf Instantiierungen von intrinsischen Eigenschaften der wahrgenommenen Gegenstände in der Außenwelt.

Subjektivismus und Objektivismus können also zwar allein aufgrund der epistemischen Modalität des Bikonditionals (BK) unterschieden werden; aber es lohnt sich, zusätzlich die möglichen Differenzen in der metaphysischen Modalität hinzuzunehmen, um drei klar voneinander differenzierte Grundpositionen hinsichtlich der Natur der Farben zu erhalten. Diese Überschneidung von metaphysischer und epistemischer Notwendigkeit kann auch die möglichen Auslegungen des Weltenbeispiels erklären. Geht man von einer Ablehnung der Notwendigkeitsthese aus, dann reicht bereits die metaphysische Modalität aus, um den Kontingenzobjektivismus und den Subjektivismus zu unterscheiden: ob also eine rigide oder eine attributive Lesart angebrachter ist. Ist die Notwendigkeitsthese jedoch gültig, dann wird (BK) automatisch metaphysisch notwendig und der Objektivismus $_{\mathrm{ACT}}$ scheidet von vorneherein aus. Nun ist ein Rückgriff auf die episte-

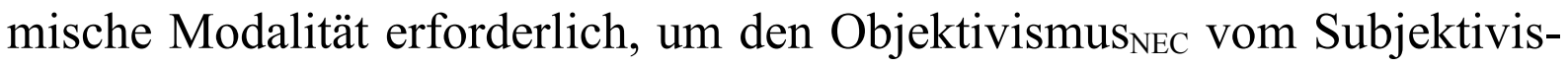
mus trennen zu können. Mehr ontologische Interpretationen des Weltenbeispieles kann es nicht geben.

\subsubsection{Die systematische Klassifikation der Theorien}

In diesem Abschnitt soll die die systematische Klassifikation der vor dem bisher dargelegten, allgemeinen Hintergrund sinnvollen Theorien der Farben entwickelt werden. Die folgenden zwei Ordnungskriterin werden dabei verwendet: (i) die Modalität des Bikonditionals (BK); (ii) die den Farben zugesprochene Eigenschaftsart. Die drei Modalitätsstufen sind bereits ausführlich diskutiert worden; im folgenden sollen deshalb nur noch kurz die 
in Frage kommenden Eigenschaftsarten aufgelistet werden.

Ganz allgemein gibt es dabei drei Möglichkeiten: die Farbeigenschaften können von den wahrgenommenen Gegenständen in der Außenwelt, vom wahrnehmenden Subjekt oder auch von beiden instantiiert werden. Zuerst einmal können Farben von Gegenständen in der Außenwelt instantiiert werden: sie können dabei primitiven, intrinsischen oder dispositionalen Status haben (wobei erst später geklärt werden soll, was Primitivität für eine Eigenschaft genau bedeutet). Ein Objektivist muß sich zwischen einer dieser drei Eigenschaftsarten entscheiden. Die dispositionale Option kann dabei als einzige in eine objektivistische und eine subjektivistische Variante aufgeteilt werden. Dann ist es auch möglich, Farben als Relationen zwischen externen Gegenständen und internen, mentalen Zuständen des Subjektes anzusehen: weswegen diese Alternative nur dem Subjektivismus offensteht. Dasselbe gilt für die beiden Arten des Projektivismus: der eine behauptet, daß Farben von internen Zuständen des Subjektes instantiiert werden, während der andere die aktuale Existenz von Farbtatsachen ganz leugnet (auch die beiden projektivistischen Varianten sollen erst in den entsprechenden Abschnitten dargestellt werden).

Mithilfe der beiden Kriteria kann nun das folgende, komplexe Schema aufgestellt werden, an dem sich die weitere Diskussion ausrichten wird. Für die allgemeine Orientierung mag es zudem später hilfreich sein, wenn die Zuordnung einzelner Philosophen zu den jeweiligen Positionen, die sie vertreten (oder zumindest zu vertreten scheinen), bereits vorweggenommen wird (der subjektivistische Relationalismus wird hier nur nebenbei erwähnt, da es keinen Vertreter dieser Position gibt).

\begin{tabular}{|c|c|c|c|}
\hline & Objektivismus $_{\mathbf{A C T}}$ & Objektivismus $_{\text {NEC }}$ & Subjektivismus $^{\text {primitiv }}$ \\
\hline intrinsisch & $\begin{array}{c}\text { Primitivismus } \\
\text { (Broackes?, J. Camp- } \\
\text { bell, Westphal) }\end{array}$ & - \\
\hline $\begin{array}{c}\text { Starker Physikalis- } \\
\text { Jackson, Jackson \& } \\
\text { Pargetter) }\end{array}$ & Starker Physikalismus & - \\
\hline
\end{tabular}




\begin{tabular}{|c|c|c|c|}
\hline$\underset{\text { (relational) }}{\text { dispositional }}$ & $\begin{array}{c}\text { Schwacher Physika- } \\
\text { lismus (Armstrong, } \\
\text { Lewis, Smart, Shoe- } \\
\text { maker) }\end{array}$ & $\begin{array}{l}\text { Schwacher Physikalis- } \\
\text { mus (Broackes?, Byr- } \\
\text { ne \& Hilbert, Hilbert) }\end{array}$ & $\begin{array}{l}\text { Subjektivistischer } \\
\text { Dispositionalismus } \\
\text { (Averill (1992), } \\
\text { Evans, Peacocke, } \\
\text { Johnston, McDo- } \\
\text { well, McGinn, } \\
\text { O'Shaughnessy?) }\end{array}$ \\
\hline intern & - & - & $\begin{array}{l}\text { Wörtlicher Projek- } \\
\text { tivismus (Boghos- } \\
\text { sian \& Velleman) }\end{array}$ \\
\hline $\begin{array}{l}\text { nicht } \\
\text { existierend }\end{array}$ & - & - & $\begin{array}{c}\text { Bildlicher Projekti- } \\
\text { vismus (Hardin, } \\
\text { Mackie, Tolliver?, } \\
\text { Wright?) }\end{array}$ \\
\hline
\end{tabular}

\subsection{Transparenz}

Eine Transparenzthese kann, ganz grob, wie folgt charakterisiert werden: sie behauptet, daß uns nicht nur bestimmte Eigenschaften in Wahrnehmungen präsentiert werden, sondern daß uns gleichzeitig diese Sinneserfahrungen auch bestimmte Merkmale oder Teile der Natur dieser Eigenschaften offenbaren. Transparenzthesen in der einen oder anderen Form spielen für nahezu jede Theorie der Farben eine besondere Rolle: ob es sich um objektivistische oder subjektivistische Positionen handelt. Im allgemeinen dienen sie, auf einer (vermeintlich) intuitiven Einsicht basierend, als Motivationsgrund für die Annahme der jeweiligen Theorie; und sie hängen, wie es sich zeigen wird, in einem engen Zusammenhang mit der durch (REP) und (PHE) beschriebenen Intuitiven Farbkonzeption. In diesem Abschnitt soll nun der enge Zusammenhang zwischen den verschiedenen Ansichten über die Transparenz der Natur der Farben einerseits und den sich darauf aufbauenden Theorien der Farben andererseits dargestellt werden. Dabei werden vier Arten von Transparenzthesen vorgestellt (der Einfachheit halber wird später nur noch eine auch wirklich diesen Namen tragen), die alle als Interpretationen zweier bestimmter Intuitionen gedeutet werden können, die wir hinsichtlich von Farbwahrnehmungen zu haben scheinen. 
Der Ausgangspunkt für die philosophische Theoretisierung dieser beiden Intuitionen stellt dabei die Debatte zwischen dem Direkten und dem Indirekten Realismus dar, auf die ich deshalb kurz zu sprechen kommen möchte. Im Anschluß daran soll der Gegensatz zwischen Objektivismus und Subjektivismus ganz allgemein dargestellt werden. Dabei wird jedoch auf die fünf prinzipiell möglichen Theorien der Farben, die bereits im Einleitungskapitel genannt worden sind, eingegangen werden. Das Kapitel über die Transparenz dient somit auch als eine Vorbereitung auf die später erfolgende Diskussion der einzelnen Positionen und soll einen ersten Überblick über mögliche Argumentationen und Schwierigkeiten verschaffen. Damit verbunden sind auch einige Vorgriffe auf noch folgende Überlegungen, die hier noch nicht ausführlich behandelt werden sollen. Es geht zunächst einmal nur um die Klärung einiger, wichtiger Punkte, die eng mit der (partiellen) Transparenz der Natur von Farben in unseren Wahrnehmungen zusammenhängen und wesentliche Differenzen zwischen den einzelnen vorgeschlagenen Alternativen hinsichtlich der theoretischen Analyse dieser Natur aufzeigen. Die Ausformulierung dieser Unterschiede und der damit verbundenen Vor- und Nachteile erfolgt erst in den nachfolgenden Kapiteln. Beginnen wir nun mit einer kurzen Darstellung der Debatte zwischen dem Direkten und dem Indirekten Realismus.

\subsubsection{Die Objektivitätsthese und die Aktualitätsthese}

Das naive Verständnis über Wahrnehmungen wird, wie gesagt, von zwei Intuitionen geprägt: der Aktualismus- und der Objektivitätsintuition. ${ }^{9}$ Die Aktualismusintuition besteht aus unserem Eindruck, daß bei jedem Auftreten einer Wahrnehmung auch eine der darin präsentierten Charakteristik entsprechende Eigenschaft tatsächlich instantiert ist. Bei einer Rotwahrnehmung scheint es so immer auch etwas „Rötliches“ zu geben. Diese Intuition diente den Indirekten Realisten als Anstoß, die Existenz von Sinnesdaten (oder Sensa) zu postulieren, die als direkt wahrgenommenen Entitäten eine Vermittlerrolle zwischen Wahrnehmung und Außenwelt einneh-

9 Dieser Abschnitt über die Debatte zwischen Direktem und Indirekten Realismus basiert im großen und ganzen auf dem noch unveröffentlichten Buchmanuskript Uncovering Appearances und den weiteren Schriften von M. G. F. Martin (vgl. z.B. Martin (2000)). 
men. Demgemäß finden sich bei ihnen Formulierungen wie die folgenden:

„Phenomenal Principle: If there sensibly appears to a subject to be something which possesses a particular sensible quality then there is something of which the subject is aware which does possess that sensible quality." (H. Robinson (1994). Perception. Routledge. London. p. 32)

„When I say „This table appears brown to me“ it is quite plain that I am acquainted with an actual instance of brownness (or equally plainly with a pair of instances when I see double). This cannot indeed be proved, but it is absolutely evident and indubitable.“ (H.H. Price (1932). Perception. Methuen. London. p. 63)

Die hierin aufgestellte Behauptung läßt sich in der Form der Aktualismusthese angeben, wobei die Eigenschaft $F$ genau diejenige sein soll, die uns in unseren Wahrnehmungen unmittelbar präsentiert wird.

(AT) Wenn ein Subjekt $S$ eine Eigenschaft $F$ unmittelbar wahrnimmt, dann gibt es auch immer etwas, daß $F$ (oder $F$-artig) ist. Das Wahrgenommenwerden eines $\mathrm{F}$ bedingt dessen Instantiierung.

Die zweite Intuition liegt dagegen dem Direkten Realismus zugrunde, demzufolge sich unsere Wahrnehmungen direkt auf Gegenstände in der Außenwelt, und nicht auf interne Zustände wie die Sinnesdaten, beziehen. Denn laut Objektivismusintuition können die von uns wahrgenommenen Eigenschaften unabhängig davon instantiiert sein, daß wir sie wahrnehmen. Haben wir zum Beispiel eine Rotwahrnehmung, dann scheint es uns intuitiv einsichtig zu sein, daß die wahrgenommenen Gegenstände auch dann ihre Farbigkeit zeigen, wenn sie gerade nicht von uns betrachtet werden. Dies scheint insbesondere deshalb für uns Gültigkeit zu haben, da wir üblicherweise die Realität um uns herum als ontologisch geistunabhängig ansehen. Die resultierende Objektivitätsthese kann wie folgt formuliert werden, wobei erneut die Eigenschaft $F$ diejenige ist, die uns in der Wahrnehmung direkt präsentiert wird:

(OT) Wenn die von einem Subjekt $S$ unmittelbar wahrgenommene Eigenschaft $F$ instantiiert ist, dann ist sie unabhängig davon instantiiert, ob sie wahrgenommen wird oder nicht. Die Instantiierung 
einer wahrnehmbaren Eigenschaft $F$ ist objektiv: sie bedingt kein Auftreten einer Wahrnehmung von sich.

Die Objektivitätsthese drückt damit die These aus, daß nur objektive, aber keine subjektiven, Eigenschaften wahrgenommen (oder repräsentiert) werden können. Viele subjektivistische Theorien der Farben gehen (vermutlich) davon aus, daß auch subjekte Farben von Farbwahrnehmungen repräsentiert werden können. Dafür, daß dies nicht stimmen kann, wird erst im Kapitel über den Farbsubjektivismus ausführlich argumentiert. Es wird sich dann auch herausstelllen, daß die Objektivitätsthese genau genommen nur behauptet, daß ein Wahrnehmungszustand nicht die Eigenschaften repräsentieren kann, die von ihm selbst ontologisch abhängig sind. Ob darüberhinaus eine stärkerer Formulierung möglich ist, soll offengelassen werden, da es für dieses Buch irrelevant ist. In jedem Fall wird in diesem Kapitel die Gültigkeit der Objektivitätsthese einfach vorausgesetzt, so daß schon von vorneherein subjektive Eigenschaften laut Direktem Realismus nicht repräsentiert werden können.

Zusammengenommen ergeben die Aktualismus- und die Objektivitätsthese nun so etwas wie eine naiv-realistische Auffassung von Wahrnehmungen. Die unmittelbar von uns wahrnehmbaren Eigenschaft $F$ sind geistunabhängig instantiiert; doch wenn eine $F$-Wahrnehmung vorliegt, gibt es auch immer die entsprechende $F$-Tatsache. Doch diese Position kann offensichtlich keine nicht-veridischen Wahrnehmungen erklären und ist somit inkonsistent. ${ }^{10}$ Aus diesem Umstand haben die Indirekten Realisten Hume und seinem Illusionsargument folgend - die Konsequenz gezogen, daß in Wirklichkeit zwei Eigenschaften im Spiel sein müssen: die direkt präsentierten Eigenschaften der Sinnesdaten und die indirekt wahrgenom-

10 Diese Inkonsistenz ergibt sich nur dann, wenn zusätzlich davon ausgegangen wird, daß veridische und nicht-veridischen Wahrnehmungen vom selben $\mathrm{Zu}$ standstypus sind (wie es durch ihre introspektive Ununterscheidbarkeit nahe gelegt wird). Disjunktivistische Wahrheitstheorien lösen das hier dargestellte Problem, indem sie diese Voraussetzung leugnen: demnach besteht ein kategorialer Unterschied zwischen korrekten und inkorrekten Sinenserfahrungen. Doch diese Position widerpricht nicht nur dem introspektiven Befund, sondern zeigt auch ihre eigenen Schwächen; zudem ist sie nur eine besondere Ausformung der intentionalistischen Theorie und kann deshalb hier vernachlässigt werden (vgl. zum Disjunktivismus: Martin (1994): 465f.; McDowell (1982)). 
menen Eigenschaften der Gegenstände in der Außenwelt. Die Aktualismusthese gilt dann nur für die unmittelbar präsentierten Eigenschaften; und der Umstand, daß die vermittels dieser wahrgenommenen, externen Eigenschaften indessen nicht immer instantiiert sein müssen, erklärt die Möglichkeit von Illusionen oder Halluzinationen.

Doch der Indirekte Realismus bringt eigene, schwerwiegende Probleme mit sich. Unter anderem kann der ontologische Status der Sinnesdaten sowie die Relationen zwischen Wahrnehmung und Sensum einerseits und zwischen Sensum und Außenwelt andererseits nicht zufriedenstellend beschrieben werden (vgl. Austin (1962); Martin (1995): 33f.). Vor allem die von der Aktualismusthese geforderte Präsentationsrelation, die eine wechselseitige, ontologische Abhängigkeit zwischen dem Auftreten von Wahrnehmungen und dem Vorliegen von Sinnesdaten miteinschließt, ist oftmals - und gerade auch im Zusammmenhang mit Farben - angegriffen worden (vgl. Armstrongs Kritik in Armstrong \& Malcom (1984): 135ff.; Armstrong (1993): 270f.; Hardin (1988): 97-108). Im Rahmen dieses Buches kann der Indirekte Realismus - und die Aktualismusthese (AT) - damit wohl als widerlegt gelten. Im folgenden werden somit der Direkte Realismus und die Objektivitätsthese (OT) als gültig angesehen werden.

\subsubsection{Die Transparenzthese und die Offensichtlichkeitsthese}

Der Direkte Realist erklärt die Möglichkeit von nicht-veridischen Wahrnehmungen nun durch das diesen zukommende Merkmal der Intentionalität (oder Repräsentationalität). Zwischen den Wahrnehmungen und den wahrgenommenen Objekten besteht eine intentionale Relation, die die Möglichkeit miteinschließt, daß das letztere Relatum - der Gegenstand nicht unbedingt immer existieren muß, damit die Beziehung generellen Bestand hat. Wie diese Repräsentationsrelation im Fall der Wahrnehmungen gedeutet werden kann, ist bereits in dem Abschnitt über den Wahrnehmungsgehalt beschrieben worden. Nun sollte der Direkte Realismus, auch wenn die Aktualismusthese in ihrer ursprünglichen Form aufgegeben worden ist, trotzdem der hinter dieser These verborgenen Aktualismusintuition gerecht werden kann. Denn auf gewisse Weise wird es sich nicht leugnen lassen, daß etwas „Rötliches“ introspektiv zugänglich ist, wenn man eine 
Rotwahrnehmung hat - ganz unabhängig davon, ob es wirklich in der Welt die entsprechende Rottatsache gibt. Damit wird der Direkte Realist den phänomenalen Charakter postulieren, der bei jeder Wahrnehmung vorhanden ist und der zudem dabei hilft, Gehaltsunterschiede aus der Sicht der ersten Person heraus feststellen zu können. Die Aktualismusintuition bezieht sich damit auf das Quale der fraglichen Wahrnehmung, welches offensichtlich immer dann instantiiert vorliegt, wenn eine entsprechende Sinneserfahrung auftritt. Die Aktualismusthese lautet also vor dem Hintergrund einer intentionalistischen Wahrnehmungstheorie:

(AT*) Wenn ein Subjekt $S$ eine Eigenschaft $F$ wahrnimmt, dann gibt es auch immer einen $F$-phänomenalen Charakter, den die fragliche Wahrnehmung zeigt. Es gibt keine $F$-Wahrnehmung ohne $F$-phänomenalen Charakter.

Die bereits entwickelte Theorie der Wahrnehmungen ist mit dieser modifizierten Aktualismusthese konform. Was passiert aber mit der Objektivitätsthese? Diese sagt ja aus, daß die wahrnehmbaren Eigenschaften $F$ unabhängig davon instantiert sind, ob sie von Subjekten wahrgenommen werden oder nicht. Zugleich ist die These (OT) jedoch durch die Introspektion gestützt, das heißt, die Eigenschaften $F$ sollten uns auf die eine oder andere Weise introspektiv zugänglich sein. Damit muß eine bestimmte Beziehung zwischen der repräsentierten Eigenschaft $F$ und dem jeweils $F$-phänomenalen Charakter der Wahrnehmungen bestehen, die zumindest die Introspektivität von Gehaltsunterschieden erlaubt. Hierbei handelt es sich wieder um das bereits erwähnte Introspektionsproblem. Die einfachste Lösungsmöglichkeit ist es nun sicherlich, auf gewisse Weise eine Identität zwischem Gehalt und phänomenalem Charakter anzunehmen. Die Idee ist es, daß, wenn wir auf Wahrnehmungen introspektieren, uns alleine repräsentationale oder repräsentierte Eigenschaften gegeben sind. Dieser Gedanke findet seinen Ausdruck in der sogenannten Transparenz- oder Diaphanitätsintuition (der Ausdruck „Transparenz“ wird von nun an nur noch speziell für diese Intuition und die entsprechende These verwendet werden): ${ }^{11}$

11 Weitere Vertreter dieser Transparenzthese sind: Evans ((1982): 230f.); Harman ((1990): 39); Dretske (1994); Armstrong (1984); sowie zumindest McGinn und Lycan. 
„The moment we try to fix attention upon consciousness and see what, distinctly, it is, it seems to vanish: it seems as if we had before us a mere emptiness. When we try to introspect the sensation of blue, all we can see is the blue: the other element is as if it were diaphanous." (Moore (1922): The Refutation of Idealism. In: Philosophical Studies. Routledge \& Kegan Paul. London. p. 22)

„If asked to focus on 'what it is like' to have this or that sort of experience, there seems to be nothing for one's attention to focus on except the content of the experience. Indeed, it may seem at first that there is nothing to focus on except the external object of perception - e.g., the tomato that one sees. Initially it may seem as though the question of what seeing the tomato is like can be none other than the question what the visually detectable aspects of the tomato are. [...] If one is asked to focus on the experience without focusing on its intentional object, or its representational content, one simply has no idea of what to do." (Shoemaker (1994b): 257)

„Focus your attention on a square that has been painted blue. Intuitively, you are directly aware of blueness and squareness as out there in the world away from you, as a features of an external surface. Now shift your gaze inward and try to become aware of your experience itself, inside you, apart from its objects. Try to focus your attention on some intrinsic feature of the experience that distinguishes it from other experiences, something other than what it is an experience of. The task seems impossible: one's awareness seems always to slip through the experience to blueness and squareness, as instantiated together in an external object. In turning one's mind inward to attend to the experience, one seems to end up concentrating on what is outside again, on external features or properties. And this remains so, even if there really is no blue square in front of one - if, for example, one is subject to an illusion. Again, one experiences blue and square as features of an external surface, but introspection does not seem to reveal any further distinctive features of the experience over and above what one experiences in undergoing the illusion.“(Tye (1995): 30)

Vor dem Hintergrund dieser Textbelege läßt sich die Transparenzthese wie folgt formulieren, wobei die Einschränkung auf bestimmte Merkmale andeuten soll, daß es durchaus immer noch möglich ist, daß uns einige Aspekte der Natur der wahrgenommenen Eigenschaften entgehen.

(TT) In der Introspektion auf Wahrnehmungen sind uns nur diejenigen objektiven und (wenn überhaupt) extern instantiierten Eigenschaften zugänglich, welche die Wahrnehmungen repräsentieren. 
Es können uns also introspektiv allein von diesen Eigenschaften (bestimmte) Merkmale gegeben sein.

Die introspektiv zugänglichen Merkmale des phänomenalen Charakters kommen somit der repräsentierten Eigenschaft zu: Gehalt und Quale fallen zusammen. Die Transparenzthese ist auch das Herzstück des Repräsentationalismus: denn ihre Gültigkeit ermöglicht, daß die Phänomenalität von Wahrnehmungen vollständig auf deren Repräsentationalität zurückgeführt werden kann. Doch sie hat noch andere, wichtige Vorteile, die ihre Attraktivität bedeutend steigern können. So löst sie nicht nur das Introspektionsproblem auf elegante Weise, sondern gibt auch eine einfache Erklärung für die Aktualitätsthese (ACT), da sie die Notwendigkeitsthese (NEC) bedingt, die wiederum (ACT) impliziert.

Zudem kann man aufgrund von (TT) ganz einfach von der Unmittelbarkeit von Wahrnehmungen ausgehen, um gar nicht erst in die Nähe eines Indirekten Realismus zu geraten. Vermittels der Repräsentationalität steht darüberhinaus auch eine Naturalisierung des phänomenalen Bewußtseins in Aussicht, dessen Erklärung somit nicht mehr ein besonderes Problem für den Materialismus darstellt. Und schließlich - für die laufende Diskussion mit am wichtigsten - motiviert und begründet die Transparenzthese den Wahrheitsanspruch der Intuitiven Farbkonzeption, da sie deren beide Aspekte miteinander auf einfachste Weise vereint: was im folgenden dargelegt werden soll. Es sei nur darauf verwiesen, daß diese unheimliche Erklärungskraft und Vereinfachung vieler Probleme einen der Transparenzthese gegenüber schnell skeptisch werden läßt; und daß sie die in sie gesetzten Hoffnungen und Erwartungen wahrscheinlich auch nicht wird erfüllen können, gerade weil damit soviel Problematisches zusammenhängt.

Die These (REP) ist so definiert worden, daß sie Farben als von Farb wahrnehmungen repräsentierte Eigenschaften ansieht, wobei eine solche Eigenschaft, neben der Repräsentierbarkeit, noch die folgenden vier introspektierbaren Merkmale aufzuweisen hat: Externalität, Objektivität, Intrinsität und Erklärungskraft. Dagegen behauptet die These (PHE), daß Farben die Phänomenalität zeigen, welche uns in der Introspektion auf Farbwahrnehmungen gegeben wird. Geht man nun, wie es die Objektivisten tun, von der These (REP) aus - daß Farben also diejenigen objektiven und externen 
Eigenschaften sind, die faktisch von Farbwahrnehmungen repräsentiert werden -, dann läßt sich aus der Transparenzthese folgern, daß auch die These (PHE) gelten muß: denn alle introspektiv zugänglichen Merkmale, auch die phänomenalen, kommen laut (TT) den repräsentierten Eigenschaften zu. Vor dem Hintergrund der Intuitiven Farbkonzeption läßt sich die Transparenzthese demnach als die Konjunktion der beiden Individuationsthesen auffassen - mit dem Zusatz, daß (REP) und (PHE) zusammen nicht unbedingt die vollständige Natur der Farben beschreiben müssen, und es aller Wahrscheinlichkeit nach auch nicht tun. Denn für den Objektivisten werden Farben voraussichtlich eine physikalisch beschreibbare Natur aufweisen (wie etwa durch die These (PHY) ausgedrückt), die von uns jedoch weder wahrgenommen, noch introspektiert werden kann. Für die Transparenzthese ergibt sich also:

(TT) Farben sind wesentlich sowohl repräsentierte als auch phänomenale Eigenschaften: das heißt, die Thesen $\left(\mathrm{IND}_{\mathrm{R}}\right)$ und $\left(\mathrm{IND}_{\mathrm{P}}\right)$ sind beide gültig und beschreiben den Farben wesentliche Merkmale.

Damit stellt die Transparenzthese auch eine Erweiterung der Objektivitätsthese (OT) dar, da die Objektivität ein Bestandteil des durch (REP) den Farben zugeschriebenen Merkmalen des repräsentationalen Aspektes des introspektiven Charakters von Farbwahrnehmungen ist. Eine weitere Konsequenz aus der Transparenzthese - zusammen mit den Aktualitätsthesen $(\mathrm{ACT})$ und $\left(\mathrm{ACT}_{\mathrm{NB}}\right)$ - ist die Notwendigkeitsthese in ihrer normalen und in ihrer über Normalbedingungen formulierten Variante: also (NEC) und $\left(\mathrm{NEC}_{\mathrm{NB}}\right)$. Denn die repräsentierte Eigenschaft und der introspektive Charakter können nur dann zusammenfallen, wenn sie notwendig miteinander korreliert sind. Dies ergibt sich auch direkt aus der zweiten Formulierung der Transparenzthese, da diese aussagt, daß die Natur der Farben den repräsentationalen und den phänomenalen Aspekt umschließt. Damit bedingen Unterschiede in der repräsentierten Farbe auch immer Unterschiede in der Phänomenalität, da diese den Farben wesentlich zukommt (und natürlich umgekehrt). Gehalt und Quale können unter keinen Umständen auseinandergerissen werden.

Die Akzeptanz der Transparenz- sowie der Notwendigkeitsthese ist je- 
doch mit einigen Schwierigkeiten verbunden. Zum einen ist es nicht klar, wie physikalisch beschreibbare Eigenschaften gleichzeitig phänomenal sein oder eine Phänomenalität vollständig erklären können, zum anderen gibt es die bekannten Gedankenexperimente, die einer notwendigen Koppelung von Quale und Gehalt entgegenstehen (zum Beispiel Jacksons knowledge-argument, oder die Fälle von Qualia-Inversion und -Abwesenheit). Auch wenn auf diese Einwände gegen den Objektivismus NEC $_{\text {erst spä- }}$ ter ausführlich eingegangen werden soll, ist es bereits hier sinnvoll, eine schwächere, aber immer noch objektivistische Alternative für eine Theorie der Farben vorzustellen: und zwar den Objektivismus ${ }_{\mathrm{ACT}}$, der die Notwendigkeitsthesen ablehnt. Da er von der Möglichkeit ausgeht, daß die repräsentierten Eigenschaften und die ihnen in der aktualen Welt entsprechenden Qualia unter anderen Bedingungen nicht mehr miteinander verbunden sein müssen, kann er auch nicht mehr die Transparenzthese aufrechterhalten. Denn wenn Gehalt und Quale auseinandergehen können, ist es nicht mehr möglich, daß die Farben sowohl die Merkmale des repräsentationalen als auch die des phänomenalen Aspektes des introspektiven Charakters von Farbwahrnehmungen zeigen können.

Die Transparenzthese (TT) wird der Objektivmus ACT $_{\text {also aufgeben }}$ müssen. Da er immer noch eine objektivistische Position darstellen sollte, ist es klar, daß nur die These (PHE, nicht aber die These (REP) fallengelassen wird: Farben bleiben objektive und repräsentierte Eigenschaften, verlieren aber die Phänomenalität als Wesensmerkmal. Die sinnliche Repräsentation der Farben ist zwar laut $\left(\mathrm{ACT}_{\mathrm{NB}}\right)$ immer noch kontingenterweise faktisch mit einer bestimmten Phänomenalität verbunden, so daß über Qualiadifferenzen auch Gehaltsdifferenzen introspektiv zugänglich bleiben; aber eine Aufspaltung der Intuitiven Farbkonzeption läßt sich nicht mehr vermeiden. Die Objektivitätsthese (OT) wird hingegen vom Objektivisten auch weiterhin aufrechterhalten.

Hinsichtlich von Farben wird jedoch oft genug ebenso ein Subjektivismus vertreten, bei dem es sich, wenn man so will, um die direkt-realistische Gegenposition $\mathrm{zu}$ den beiden Varianten des Objektivismus handelt. Es ist wichtig, daraufhinzuweisen, daß ein Farbsubjektivismus immer noch mit objektivistischen Positionen hinsichtlich von anderen Eigenschaftsklassen (wie etwa Formen) konsistent ist, so daß die folgende Diskussion sich auf 
Farbwahrnehmungen beschränkt und nur durch weitere Annahmen auch auf andere Eigenschaftsarten (zum Beispiel andere sekundäre Qualitäten) ausgeweitet werden kann. Ein Farbobjektivismus wird dagegen im allgemeinen auch Objektivist hinsichtlich der meisten anderen, wahrnehmbaren Eigenschaften sein wird. Der Farbsubjektivismus muß nun offensichtlich die Objektivität der Farben leugnen und somit die Objektivitätsthese (OT) ablehnen. Gleichzeitig hält er auch nicht die Aktualismusthese (AT) aufrecht, da er auf einer intentionalistischen Wahrnehmungstheorie, und keiner representativen Sinnesdatentheorie, fundiert ist.

Die Ablehnung der Objektivitätsthese bedingt auch die Zurückweisung der Individuationsthese (REP) und der Transparenzthese (TT), da beide Farben wesentlich als objektive Eigenschaften ansehen. Damit entgeht der Subjektivismus gleichzeitig den Schwierigkeiten, denen sich ein Befürworter der Transparenzthese ausgesetz sieht. Der Subjektivismus wählt also einen anderen Weg als noch der Objektivmus ${ }_{\text {Aст }}$ : er sieht die Farben als phänomenale Eigenschaften an und behält somit (PHE) bei. Es ist dem Subjektivisten daneben wahrscheinlich noch nicht einmal möglich, zumindest die Repräsentierbarkeit von Farben weiterhin zu behaupten (unsere Farbwahrnehmungen repräsentieren somit andere, physikalische Eigenschaften in der Welt). Denn als ein Vertreter des Direkten Realismus (der sich zudem kaum einer externalistischen Gehaltstheorie wird entziehen können) werden für ihn als repräsentierte Eigenschaften für visuelle Wahrnehmungen nur objektive Reize in der Außenwelt in Frage kommen: geistabhängige Eigenschaften können sinnvollerweise nicht repräsentiert werden. ${ }^{12}$ Mit der Aufgabe der Objektivität und der Repräsentierbarkeit ist auch der Verzicht auf das Merkmal der Externalität verbunden: es mach gar keinen Sinn, Farben als phänomenale und nicht-repräsentierte Eigenschaften anzusehen und sie gleichzeitig doch als in der Außenwelt instantiiert anzunehmen. Nur ein subjektivistischer Dispositionalismus (oder Relationalismus) kann eventuell die Repräsentierbarekeit, und damit auch die Erklärungskraft und die Intrinsität, aufrechterhalten. ${ }^{13}$

12 Für diese These wird zu Anfang des Kapitels über den Subjektivismus argumentiert. Bis dahin wird sie einfach als gültig vorausgesetzt.

13 Interessanterweise macht die These (NEC) mit der Aufgabe von (IND $)$ für einen Subjektivisten keinen Sinn mehr, bezieht man sie auf Farben: wenn es keinen Farbgehalt gibt, kann dieser auch nicht mehr mit dem Farbquale notwendigerwei- 
Nun stellt es sich aber heraus, daß - allein auf Farben bezogen - der Subjektivismus im Grunde als eine Art von direkt-realistischer Spielart des Indirekten Realismus aufgefaßt werden kann. Während der eben beschriebene Farbobjektivismus nichts anderes als die These vertritt, daß Farb wahrnehmungen objektive Farben in der Welt auf eine direkte, intentionale Weise repräsentiert, so weichen sowohl der Indirekte Realismus als auch der Subjektivismus dieser Position aus (und zudem aus ähnlichen Gründen). Für die Sinnesdatentheorien sind Farben, wie alle sekundären Qualitäten, zuallererst Eigenschaften der Sensa, die direkt wahrgenommen werden. Dagegen sind die indirekt repräsentierten Eigenschaften in der Welt primäre Qualitäten, das heißt, physikalische Eigenschaften. Deswegen macht es auch Sinn, zu behaupten, daß letztere Art von Qualitäten nicht wirklich (unmittelbar) sichtbar oder wahrnehmbar sind, sondern auf ihre Existenz nur mithilfe der ersteren Art von Qualitäten geschlossen werden kann. Der Farbsubjektivismus ist im im prinziellen, strukturellen Aufbau eine ganz ähnliche Position, da er Farben als interne, nicht-repräsentierbare Eigenschaften ansehen muß, die auf die wahrgenommenen, physikalischen Tatsachen in der Welt auf die eine oder andere Weise projeziert werden. Nur werden die Farben diesmal nicht als Eigenschaften von Sinnesdaten, sondern von anderen, subjekt-internen Trägern angesehen: und zwar entweder von cerebralen oder von mentalen Zuständen.

Dahinter verbirgt sich eine weitere Parallelität beider Theorien. Denn in gewissem Sinne vertraut auch der Subjektivismus einer Variante der Aktualismusthese, derzufolge bei jeder Farbwahrnehmung immer auch eine für die Projektion der Farbigkeit auf die Welt verantworliche Tatsache vorliegt - ganz unabhängig davon, ob auch die repräsentierte, physikalische Eigenschaft in der Welt instantiiert ist. Zudem, da Farben nicht repräsentiert werden, sondern die für die gesehene Farbigkeit allein verantwortlichen Tatsachen immer mit den Wahrnehmungen zusammen auftreten, wird es vermutlich auch eine wechselseitige, ontologische Abhängigkeit zwischen diesen Farbtatsachen (wenn man sie so nennen will) und den ent-

se verbunden sein. Auch die ganze Debatte über Qualia-Inversion mit Farbeigenschaften als Bezugspunkt ist aus subjektivistischer Sicht nicht mehr intelligibel. Natürlich kann weiterhin trotzdem eine solche Debatte mit Hinsicht auf Reflektanzeigenschaften geführt werden; und hinsichtlich dieser Eigenschaften läßt sich auch noch die These (NEC) ablehnen oder annehmen. 
sprechenden Farbwahrnehmungen geben. Denn wenn laut (PHE) allein das Quale oder die damit immer verbundenen interne Tatsache über die präsentierte, gesehene Farbe entscheidet, und wenn diese Farbtatsache geistabhängig sein soll, dann kann es wohl kaum vorkommen, daß Farbe oder Farbwahrnehmung unter irgendwelchen Umständen voneinander abweichen. Nur durch die stipulative Einführung von Normalbedingungen könnte dies geändert werden. Doch über diese Punkte wird erst der Abschnitt über den Subjektivismus Klarheit schaffen können. Zunächst erlaubt siese Nähe zum Indirekten Realismus erst einmal die folgende Formulierung der Aktualismusthese für den Farbsubjektivisten:

(AT**) Wenn ein Subjekt $S$ eine Eigenschaft $F$ wahrnimmt, dann ist auch immer eine subjektive, $F$-phänomenale Eigenschaft instantiiert.

Mögliche Kandidaten für diese Eigenschaften sind etwa sinnliche Eigenschaften des Gesichtsfeldes oder auch die Farbqualia selbst (im letzteren Fall würden (AT*) und (AT**) wieder zusammenfallen). Die Transparenzintuition bleibt für den Subjektivismus ebenfalls in gewisser Weise erhalten (wie der Objektivismus die Aktualismusintuition nicht vollständig aufgeben kann). Dahinter verbirgt sich die subjektivistische Auffassung, daß nicht nur die Instantiierung von Farbtatsachen ontologisch geistabhängig sind, sondern daß das Subjekt auch zudem in der Lage ist, die Natur der Farbeigenschaften allein durch Introspektion auf seine Farbwahrnehmungen vollständig epistemisch zu erfassen, weil diese Natur uns bereits in den Sinneserfahrungen selbst in gewissem Sinne transparent gegeben ist. Um Verwechslungen mit der recht ähnlichen Transparenzthese zu vermeiden, werde ich die betreffende These, Johnston folgend, Offensichtlichkeitsthese nennen (revelation). Hier sind einige Formulierungen dieser These, wie sie sich bei Subjektivisten (oder deren Kritikern) finden lassen, aber auch bei Philosophen wie Russell, die in einem Abschnitt ihres Lebens Sinnesdatentheoretiker gewesen sind:

„Color words are words for properties which are of such a kind that their whole and essential nature as properties can be and is fully revealed in sensory-quality experience given only the qualitative character that that experience has." (Strawson (1989): 224) 
„The particular shade of colour that I am seeing... may have many things to be said about it. [...] But such statements, though they make me know truths about the colour, do not make me know know the colour itself better than I did before: so far as concerns knowledge of the colour itself, as opposed to knowledge of truths about it, I know the colour perfectly and completely when I see it and no further knowledge of it itself is even theoretically possible." (Russell (1912): 47)

„The intrinsic nature of canary yellow is fully revealed by a standard visual experience as of a canary yellow thing. [...] One naturally does take and should take one's visual experience as of, e.g. a canary yellow surface, as completely revealing the intrinsic nature of canary yellow, so that canary yellow is counted as having just those intrinsic and essential features which are evident in an experience as of canary yellow. Hence, canary yellow is a simple non-relational property pervading surfaces, volumes and light sources. [...] Revelation tells us that the natures of the colors are, in George Harding's useful idiom, laid bare in visual experience. The nature of canary yellow is supposed to be fully revealed by visual experience so that once one has seen canary yellow there is no more to know about the way canary yellow is. Further investigation and experience simply tells us what further things have the property and how that property might be contigently related to other properties." (Johnston (1992): 138f.)

Die hier beschriebene These ist tatsächlich eine radikalere Variante der Transparenzthese, da sie zwar die Auffassung beibehält, daß wahrgenommenen Eigenschaften (wie hier die Farben) genauso sind, wie sie uns erscheinen, aber das zusätzliche Zugeständnis in (TT) - daß ein Teil der Natur uns nicht zugänglich sein muß - strikt ablehnen: unsere Wahrnehmungen - und bereits jede einzelne für sich genommen - offenbaren uns die ganze Wahrheit über die Natur der Farben. Natürlich gibt es Wahrheiten über Farben, die uns nicht schon in einer einzigen, introspektierbaren Wahrnehmung, oder überhaupt nicht in Wahrnehmungen, gegeben sind, aber diese betreffen nicht die wesentlichen Merkmale der Farben, sondern nur kontingente Fakten über diese: welche Objekte zum Beispiel als ihre Instanzen fungieren. Aber die durch Introspektion auf Sinneserfahrungen zugänglichen Merkmale von Farben erschöpfen die Natur derselben. Damit ergibt sich also für die Offensichtlichkeitsthese:

(OS) Die Natur der Farben ist ganz genauso, wie sie uns in Farbwahrnehmungen erscheint. Das heißt, alle wesentlichen Merkmale - 
und nur diese - sind uns in unseren Farbwahrnehmungen gegeben oder präsentiert.

Die Offensichtlichkeitsthese ist somit, wenn man sie wir hier allein für den Fall der Farben betrachtet, die Konjunktion der beiden Individuationsthesen (REP) und (PHE), da diese beiden zusammen alle Merkmale des introspektiven Charakters von Farbwahrnehmungen den Farben wesentlich zuschreiben: Farben sind nichts anderes als repräsentierte und phänomenale Eigenschaften. Doch es gibt, wie bereits oben erwähnt, ein offenkundiges Problem mit den in (REP) enthaltenen Merkmalen der Objektivität und der Repräsentierbarkeit, welches den Subjektivisten schließlich zur Aufgabe von (REP) zwingen wird. Die Objektivität kann der Subjektivismus natürlich nicht aufrechterhalten: für ihn bestehen Farbtatsachen immer nur geistabhängig. Doch können für den Subjektivismus (mit der eventuellen Ausnahme der dispositionalistischen Variante) aller Voraussicht nach Farben auch nicht repräsentiert sein. Ein Grund hierfür ist, daß keine objektiven Tatsachen über die wahrgenommene Farbigkeit der Gegenstände in der Welt als Wahrheitsbedingungen in Frage kommen. Ein Art von Nicht-Veridizität kann nur stipulativ auf Tatsachen über wahrnehmende Subjekte innerhalb einer Gemeinschaft fundiert werden. Denn die Farbwahrnehmungen der Subjekte dienen als einziger Maßstab für die Bewertung der eventuellen Korrekt- oder Inkorrektheit, da Farben selbst keine objektive Natur besitzen.

Damit ist für den Subjektivisten auch die Intrinsität uninteressant geworden, da diese nur im Zusammenhang mit der Externalität und der Repräsentierbarkeit von Farben Sinn macht (natürlich können die ,internen“, subjektiven Farben möglicherweise ebenfalls intrinsische Eigenschaften von bestimmten Zuständen sein, aber die Intrinsität bezieht sich immer auf die externen, wahrgenommenen Gegenstände). Die Externalität ist natürlich ebenfalls aufgegeben, da die nicht-repräsentierten Farben intern instantiiert sein müssen (vgl. auch ( $\left.\mathrm{AT}^{* *}\right)$ ). Die Erklärungskraft wird als einzige vielleicht erhalten bleiben, da der Umstand der Projektion einer Farbigkeit auf die Welt immer noch durch die internen Farbeigenschaften erklärt werden kann. Doch dann wird sie sich prinzipiell von der eingangs definierten Erklärungskraft unterscheiden, weil sie aufgrund der These 
$\left(\mathrm{AT}^{* *}\right)$ eigentlich nur noch eine Trivialität ausdrückt. In diesem Sinne ist es ratsam, davon zu sprechen, daß auch die Erklärungskraft nicht mehr gegeben ist. Damit muß der Subjektivismus die These (REP) komplett aufgeben. Was ihm natürlich bleibt, ist die These (PHE): Farben sind wesentlich phänomenale Eigenschaften.

Es könnte vielleicht angezweifelt werden, ob die Merkmale von dem repräsentationalen Aspekt tatsächlich Merkmale des introspektiven Charakters von Farbwahrnehmungen sind und somit zu unserer common-senseAuffassung von Farben gehören. Doch wie Jackson argumentiert, ist es wahrscheinlich viel zweifelhafter, daß die These (OS) ein Bestandteil unserer gewöhnlichen Ansicht über Farben sein soll, da wir nicht unbedingt glauben (müssen), daß die Natur wahrnehmbarer Eigenschaften von uns vollständig sinnlich erfahrbar ist (vgl Jackson (1996): 210ff.). Deswegen kommt die Transparenzthese (TT) unserer intuitiven Auffassung auch näher und sollte als angemessenste Position angestrebt werden. In jedem Fall ist es für einen Subjektivismus unmöglich, die These (REP) aufrechtzuerhalten: somit reduziert er im Grunde die Offensichtlichkeitsthese (OS) auf die These (PHE) und muß - wie auch der Objektivismus ACT $_{-}$- eine Aufspaltung der Intuitiven Farbkonzeption postulieren. Es ist zudem fraglich, ob überhaupt alle Subjektivisten die Gültigkeit (REP) von Anfang an behaupten wollen. Strawson zum Beispiel spricht explizit nur von dem qualitativen Charakter von Farbwahrnehmungen: wenn er damit nicht den gesamten introspektiven Charakter gemeint hat, so trifft ihn die vorherige Argumentation nicht, da er ihr von vorneherein aus dem Weg geht. Trotzdem kann jeder Subjektivist dementsprechend nur die folgende, schwächere Variante der Offensichtlichkeitsthese vertreten (die für sich genommen mit (PHE) identisch ist):

(OS*) Die phänomenale Natur der Farben ist ganz genauso, wie sie uns in Farbwahrnehmungen erscheint. Das heißt, (PHE) ist wahr.

Dabei ist es nun wieder möglich geworden, daß es noch zusätzliche Wesensmerkmale von Farben geben kann: dann nämlich, wenn Farben nicht mit den Farbqualis gleichgesetzt werden, sondern als dispositionale oder relationale Eigenschaften der externen Gegenstände, oder auch als sinnli- 
che Eigenschaften des Gesichtsfeldes, angesehen werden: in diesen Fällen ist es nicht klar, ob die gesamte Natur der Farben durch die uns zugänglichen, phänomenalen Merkmale erschöpt wird. Auch Johnston kommt zu dem Schluß, daß (OS) abgeschwächt werden muß (vgl. Johnston (1992): 142). Er sieht darin auch den Entschluß der Projektivisten begründet, nicht mehr von externen, repräsentierten Farben zu sprechen und, im Extremfall, die Existenz von Farbtatsachen ganz zu leugnen (vgl. Johnston (1992): Fn. 6). Etwas anders mag es für den von Johnston vertretenen nichtphysikalistischen Dispositionalismus (sowie den Relationalismus) aussehen, der eine Sonderolle unter den subjektivistischen Positionen einnimmt, weil er sich dem Objektivismus in vielem annähert und als eine Art von Mischtheorie gelten kann. Johnston hofft selbst auf die Möglichkeit, mithilfe einer dispositionalistischen Theorie wenigstens einige der Merkmale des repräsentationalen Aspektes für die subjektiven Farben retten zu können (wie etwa die Repräsentierbarkeit oder die Externalität). Darüberhinaus führt er ein weiteres, ganz anderes Argument gegen die Aufrechterhaltung der Offensichtlichkeitsthese durch den subjektivistsiche Dispositionalisten an, welches auch gegen die Aufrechterhaltung der abgeschwächten Version (OS*) ohne den Zusatz weiterer, nicht-wahrnehmbarer Wesensmerkmale von Farben spricht.

„The decisive consideration is... that steady colors, opposed say to highlights, do not appear to be relational properties and hence do not appear to be dispositions to look colored. A basic phenomenological fact is that we see most of the colors of external things as 'steady' features of those things, in the sense of features which do not alter as the light alters and as the observer changes position. (This is sometimes called 'color constancy'.) A course of experience as of the steady colors is a course of experience as of light-independent and observer-independent properties... Contrast the highlights: a course of experience as of the highlights reveals their relational nature. They change as the observer changes position relative to the light source. They darken markedly as the light source darkens. With sufficiently dim light they disappear while ordinary colors remain. [...] Thus there is some truth in the oft-made suggestion that (steady) colors don't look like dispositions; to which the natural reply is 'Just how would they have to look if they were to look like dispositions?'; to which the correct response is that they would have to look like colored highlights or better, like shifting, unsteady colors, e.g. the swirling evanescent colors that one sees on the back of compact discs." (Johnston (1992): 141) 
Es sollte klar sein, daß die Beispiele, die Johnston anführt, eher als Farbillusionen anzusehen sind denn als genuine Farben (wie etwa auch holographische Effekte oder farbig reflektierende Ölschlieren). Dies wird besonders im Zusammenhang mit der Erkenntnis deutlich werden, daß die Farbigkeit von Licht oder Lichtquellen ebenfalls im Grunde eine Fehlrepräsentation darstellt, da die betreffenden Entitäten eigentlich keine Farbträger sein können, weil unser Wahrnehmungsapparat sich nicht primär zur Detektion ihrer möglichen Farbigkeit evolutionär entwickelt hat (vgl. Zeki (1993): 238). Trotzdem kann man den phänomenologischen Unterschied, auf den er aufmerksam machen möchte, ohne weiteres bemerken. So wie uns die physikalische Natur von Eigenschaften in der Welt nicht sinnlich gegeben sein kann, so gilt dies auch für die dispositionale Natur von „beständigen“, nicht-schimmernden Farben. Auch Smith argumentiert, daß gerade der subjektivistische Dispositionalist (OS*) - oder (OS) - nicht so verstehen kann, daß der Umstand, daß Farben als über Farbwahrnehmungen als Wirkungen analysierte Dispositionen zu gelten haben, uns sinnlich präsentiert ist oder sein kann; vielmehr handelt es sich hierbei um philosophische oder quasi-theoretische Überlegungen (vgl. Smith (1993): 274); ferner vertreten Boghossian und Velleman als Projektivisten diese Meinung (vgl. (Boghossian \& Velleman (1989): 86ff.). Doch trotzdem hat die von Johnston (und auch anderen) vertretene Variante des Subjektivismus - der nicht-physikalistische Dispositionalismus - dem Projektivismus gegenüber eventuell den Vorteil, bis zu einem gewissen Grad die repräsentationalistischen Merkmale für Farben beibehalten zu können.

Der Primitivismus ist interessanterweise hinsichtlich seiner Auffassung über die Natur von Farben von dem Subjektivismus gar nicht so weit entfernt. Dadurch nimmt er, wie der Dispositionalismus für den Subjektivisten, eine Sonderrolle für den Objektivisten ein. Denn es ist ebenfalls das erklärte Ziel der primitivistischen Position (wie sie etwa John Campbell vorgeschlagen hat), der Offensichtlichkeitsthese (OS) gerecht zu werden; und das heißt auch, die objektivistische Transparenzthese (TT) von der Möglichkeit eines Zusatzes (wie einer Identifikation der Farben mit physikalisch beschreibbaren Eigenschaften) zu befreien. Im Grunde ist der Primitivismus bemerkenswerterweise die einzige Theorie der Farben, die 
ernsthaft den Anspruch vertreten kann, einerseits der Intuitiven Farbkonzeption vollkommen gerecht $\mathrm{zu}$ werden und andererseits möglicherweise ohne weitere, analysierende Zusatzthesen über die Natur der Farben auskommen zu können. In seinem Text $A$ Simple View of Colour gibt Campbell eine Formulierung der (von ihm „Transparenzthese“ genannten) Offensichtlichkeitsthese, die er mit Bezug auf Farben vertritt:

\begin{abstract}
„The perception reveals the whole character of the [perceived] property to us. [...] This is not a kind of physicalism about colours. To suppose that it must be is to assume an identification of the physical and the objective which the thesis may question. It may instead be that the characters of the colours are simply transparent to us. Of course, we often have to consider cases in which the character of a property is not transparent to us; but there may also be cases in which transparency holds. [...] The real nature of the property [redness] is... transparent to us." (J. Campbell (1993): 257f.)
\end{abstract}

Inwiefern der Primitivismus eine plausible Theorie der Farben ist, soll erst später diskutiert werden. Diese Diskussion wird auch zu einer Einschätzung der Plausibilität davon führen, ob es für den Primitivisten wirklich möglich ist, die starke Offensichtlichkeitsthese aufrechtzuerhalten.

\title{
3.2.3. Zusammenfassung der Debatten
}

In diesem Kapitel sind bisher - jeweils anhand verschiedener Ausformungen der Transparenzintuition - die beiden Debatten zwischen den Direkten und den Indirekten Realisten hinsichtlich einer angemessenen Wahrnehmungstheorie sowie zwischen dem Objektivismus und dem Subjektivismus hinsichtlich der Natur und dem ontologischen Status von Farben besprochen worden. Ein direkter Zusammenhang läßt sich einerseits darin sehen, daß die die jeweiligen Diskussionen beherrschenden vier Thesen sehr ähnlich sind und auf die eine oder andere Weise als „Transparenzthesen“ gelten können, weil sie einen Teil der Natur der Farben als in Farbwahrnehmungen sinnlich gegeben betrachten; und daß andererseits der Indirekte Realismus und der Subjektivismus sich in der Entgegensetzung gegen einen objektivistischen Direkten Realismus mit Bezug auf Farbwahrnehmungen eine vergleichbare Rolle einnehmen. 
Während nun der Direkte Realismus sich hauptsächlich auf die Objektivitätsintuition stützt, basiert die Annahme des Indirekten Realismus auf der Aktualismusintution: erstere behauptet die Geistunabhängigkeit der wahrgenommenen Tatsachen, wogegen letztere aussagt, daß bei jeder Wahrnehmung einer Eigenschaft auch eine entsprechende, unmittelbar wahrgenommene Tatsache existieren muß. Da beide Intuitionen, zusammen mit der Möglichkeit von nicht-veridischen Wahrnehmungen (die zudem von veridischen introspektiv nicht unterscheidbar sind), inkonsistent sind, muß eine der beiden aufgegeben werden. Gegen die Sinnesdatentheorie gerichtete Argumente und Überlegungen legen es nahe, den Intentionalismus zu bevorzugen, die Aktualismusthese (AT) aufzugeben und die Objektivitätsthese (OT) beizubehalten. Vor dem Hintergrund des Direkten Realismus lassen sich nun die möglichen Farbtheorien genauer charakterisieren. Dabei bleiben sowohl die Aktualismus- als auch die Objektivitätsintuition in der einen oder anderen Form erhalten.

Die Grundlage des Objektivismus bildet die These (REP), derzufolge den Farben die Merkmale des repräsentationalen Aspektes zukommen und die die These (OT) direkt impliziert. Mithilfe des introspektiven Charakters von Farbwahrnehmungen läßt sich auch die Aktualismusintuition erklären, indem die These ( $\left.\mathrm{AT}^{*}\right)$ vertreten wird, die für veridische wie für nicht-veridische Farbwahrnehmungen gleichermaßen Farbqualia annimmt. Damit ist bereits - vor dem Hintergrund der Aktualitätsthese $\left(\mathrm{ACT}_{\mathrm{NB}}\right)$ - der Objektivismus $_{\mathrm{ACT}}$ beschrieben. Der Objektivismus ${ }_{\mathrm{NEC}}$ kann mithilfe der Notwendigkeitsthese $\left(\mathrm{NEC}_{\mathrm{NB}}\right)$ zusätzlich die These (PHE) akzeptieren, so daß Farben vielleicht auch eine diesen wesentliche Phänomenalität zugeschrieben werden kann. Dies ermöglicht zudem die Akzeptanz der Transparenzthese (TT), die aussagt, daß die Farben wirklich so sind, wie sie uns in Farb wahrnehmungen erscheinen. Ein Teil der Natur der Farben ist uns jedoch verborgen: dieser wird durch eine empirisch gestützte These (PHY) über die Identität von Farben mit bestimmten physikalisch beschreibbaren Eigenschaften erfaßt werden.

Der Primitivismus versucht sogar, auf diese zusätzliche These zu verzichten, indem er Farben als primitive, natürliche Arten ansieht. Damit kann er vielleicht die Transparenzthese durch die Offensichtlichkeitsthese (OT) ersetzen, die die Natur der Farben in den Merkmalen des introspektiven Charakters von Farbwahrnehmungen erschöpft sieht: Farben sind phä- 
nomenale und repräsentierte Eigenschaften, nicht mehr und nicht weniger. Es gelten (REP) und (PHE) - und diese benennen bereits alle Wesensmerkmale der Farben. Die Motivation für die Offensichtlichkeitsthese liegt darin, da sie genau dem naiven Verständnis von Farben entspricht und weder etwas hinzufügt, noch Abstriche davon macht (damit gleicht sie der Konjunktion der Aktualismus- und der Objektivitätsthese in dem naiv-realistischen Verständnis von Wahrnehmungen). Doch die common-sense-Auffassung, daß Farben nicht-physikalische, objektive, repräsentierte und phänomenale Eigenschaften sind - wie es die Offensichtlichkeitsthese fordert kann höchstens vom Primitivismus ganz miteingeschlossen werden, so daß dieser, ohne weitere Zusatzüberlegungen, in gewissem Sinne als eine „ideale“ Position gelten kann.

Nun ist es aber fraglich, ob der Primitivismus tatsächlich eine plausible Farbtheorie darstellen kann. Deswegen ist es sinnvoll, auf die Möglichkeiten hinzuweisen, diese Position abzuschwächen. Die objektivistischen Alternativen sind bereits erwähnt worden: zum einen wird die These (PHY) hinzugenommen, so daß Farben ihren primitiven Status verlieren; zum anderen wird die Offensichtlichkeitsthese durch weniger starke Varianten durch die Transparenz- oder sogar durch die Objektivitätsthese - ersetzt. $\mathrm{Da}$ es (unabhängig von der Plausibilität des Primitivismus) zudem nicht der Fall zu sein scheint, daß die Offensichtlichkeitsthese (OS) aufgrund ihres Vollständigkeits- oder Erschöpfungsanspruches ganz unserer naiven Auffassung entspricht (vgl. Jackson (1996): 211), ist es vielleicht sinnvoller, den Objektivismus $\mathrm{NEC}_{\mathrm{N}}$ zusammen mit der Transparenzthese als die bestmöglichste Theorie anzusehen, da diese nicht nur die Intuitive Farbkonzeption aufrechterhält, sondern auch Freiraum dafür läßt, daß dieser noch nicht die ganze Natur der Farben charakterisiert. Alle anderen Farbtheorien (außer vielleicht dem Primitivismus) müssen dem Objektivismus ${ }_{\mathrm{NEC}}$ gegenüber Konzessionen machen.

Kann diese Position also plausibel und konsistent gemacht werden, dann wird es sich kaum mehr lohnen, noch mögliche Alternativen zu betrachten (gerade hierin liegt auch die enorme, intuitive wie theoretische Anziehungskraft des Repräsentationalismus begründet, der einen Objektivismus $_{\mathrm{NEC}}$ mit sich bringt). Und außerdem kann es vielleicht als Vorteil des Objektivismus $_{\mathrm{NEC}}$ gegenüber dem Primitivismus gelten, daß ersterer die Wissenschaft der Farben miteinzuschließen vermag. Der Primitivismus ist 


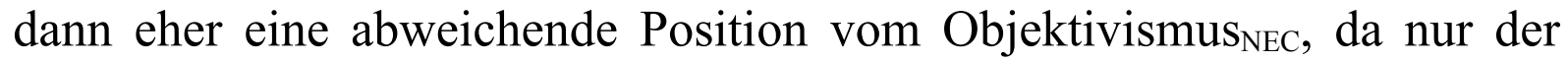
Phänomenalität, nicht aber der Physik gerecht wird. Dies hängt ganz davon ab, wie das Hauptproblem für die Theorien der Farben interpretiert wird: Erscheinung und Wirklichkeit von Farben zusammenzubringen. Setzt man einen wissenschaftlichen Realismus, dann scheint der Objektivismus $\mathrm{NEC}_{\mathrm{NEC}}$ tatsächlich die bessere Lösung für dieses Problem zu sein, da es alles andere als klar ist, ob primitive, nicht physikalisch analysierbare Eigenschaften in ein naturwissenschaftliches Weltbild integriert werden können. Vor diesem Hintergrund sollte demnach eigentlich der Objektivismus $\mathrm{SEC}_{\mathrm{NEC}}$ als ideale und erstrebenswerteste Theorie der Farben gelten.

Der Subjektivismus wird hingegen die Objektivitätsthese (OT) natürlich leugnen müssen: damit ist die Möglichkeit einer systematische Veridizität bereits nicht mehr gegeben. Das führt auch zu einer Ablehnung von (REP), so daß Farben - mittels (PHE) - nur als phänomenale Eigenschaften angesehen werden. Damit verbunden ist nicht nur die entsprechende Abschwächung der Offensichtlichkeitsthese zu (OS*), sondern auch ein anderer Umgang mit der Aktualismusintuition: (AT**) weitet den Kandidatenkreis für die immer mit Farbwahrnehmungen verbundenen phänomenalen Eigenschaften aus (zum Beispiel auf Eigenschaften des visuellen Gesichtsfeldes). Der wörtliche Projektivismus wird Farben mit letzteren Eigenschaften gleichsetzen; der bildliche oder eliminative Projektivismus zieht aus der Widerlegung der These (OS) dagegen die Konsequenz, daß es faktisch gar keine instantiierten Farben gibt. Als eine besondere Variante des Subjektivismus versucht der nicht-physikalistische Dispositionalismus (oder auch der Relationalismus), Farben wenigstens teilweise als Eigenschaften externer Gegenstände retten und somit möglicherweise bis auf die Objektivität alle Merkmale des durch (REP) beschrieben repräsentationalen Aspektes für Farben erhalten zu können. Es ist jedoch gerade mit Bezug auf die Repräsentierbarkeit fraglich, ob dies gelingen wird: denn da der Direkte Realismus auf der Objektivitätsthese basiert, ist es zu erwarten, daß nur objektive Tatsachen repräsentiert werden können.

Die fünf eingangs in der Einleitung erwähnten Theorien der Farben, die alle den Direkten Realismus hinsichtlich von Farbwahrnehmungen akzeptieren, lassen sich nun - anhand ihrer, zumeist selbstgewählten, Erklärungsansprüche und den infolgedessen ihre Position ausmachenden Thesen - wie folgt in einer Tabelle systematisieren. Darüber, ob die einzelnen 
Theorien die ihnen zugesprochenen Thesen auch tatsächlich aufrechterhalten können werden, ist damit natürlich noch nichts ausgesagt worden. Insbesondere wird für den (hier allein subjektivistisch verstandene) Dispositionalismus die Akzeptanz der These (REP) problematisch sein (wobei er das darin enthaltene Objektivitätsmerkmal sicherlich von vorneherein nicht beibehalten möchte); und der Objektivismus ${ }_{\mathrm{NEC}}$ wird wahrscheinlich Schwierigkeiten mit der Phänomenalität (PHE) der Farben bekommen. Außerdem sollte noch einmal darauf hingewiesen werden, daß ein Repräsentationalismus, nicht nur den Objektivismus, sondern auch entweder die Transparenzthese (TT) oder die Offensichtlichkeitsthese (OS) voraussetzt. Dementsprechend kann ein Repräsentationalist nur zwischen dem Objektivismus ${ }_{\mathrm{NEC}}$ und dem Primitivismus wählen; alle anderen Positionen bedingen eine phänomenalistische Theorie des phänomenalen Charakters von Wahrnehmungen. Doch hier nun die Zuordnung der einzelnen Farb- und Transparenzthesen (im weiten Sinne) zu den fünf möglichen Analysen der Natur der Farben:

\begin{tabular}{|c|c|c|c|}
\hline & $\left(\right.$ IND $\left._{\mathbf{R}}\right) /($ REP) & $\left(\right.$ IND $\left._{\mathbf{P}}\right) /($ PHE) & Transparenz \\
\hline Objektivismus $_{\mathrm{ACT}}$ & ja & nein & $(\mathrm{OT})$ \\
\hline Objektivismus $_{\mathrm{NEC}}$ & $\mathrm{ja}$ & ja & $(\mathrm{TT})$ \\
\hline Primitivismus & ja & ja & $(\mathrm{OS})$ \\
\hline Dispositionalismus & (ja) & ja & $\left(\mathrm{OS}^{*}\right)$ \\
\hline Projektivismus & nein & ja & $\left(\mathrm{OS}^{*}\right)$ \\
\hline
\end{tabular}




\subsection{Normalbedingungen}

Der Begriff der Normalbedingung hängt eng mit dem der repräsentationalen Veridizität zusammen. Denn es ist festgelegt worden, daß eine Wahrnehmung genau dann repräsentational veridisch ist, wenn sie unter Normalbedingungen auftritt. ${ }^{14}$ Weitere Erläuterungen $\mathrm{zu}$ diesem Thema sind bisher jedoch ausgeblieben, was in diesem Abschnitt aber nachgeholt werden soll. Einige, wichtige Fragestellungen sind dabei $\mathrm{zu}$ beachten: wie Normalbedingungen überhaupt bestimmt werden können; ob sie konkret für den Fall von Farbwahrnehmungen formuliert werden können, und wenn ja, wie sie dann genau aussehen werden; und schließlich, ob es auch Theorien der Farben gibt, die ganz ohne Normalbedingungen auskommen können. ${ }^{15}$ Die folgenden Abschnitte werden sich dabei fortwährend auf die Erkenntnisse der wissenschaftlichen Erforschung der Farben berufen (vgl. Zeki (1993); Hardin (1988): Kap. 1).

Diese Untersuchungen über die dem Farbensehen zugrundeliegenden physikalischen und neurophysiologischen Prozeße haben nun gezeigt, daß man davon ausgehen kann, daß unsere Farbwarhrnehmungen bestimmte Reflektanzeigenschaften detektieren. Ob es sich dabei um Reflektanzprofile oder -typen handeln wird, ist dabei für Frage nach den Normalbedingungen von geringerer Bedeutung. Das Reflektanzprofil einer (homogen gefärbten) Oberfläche bestimmt sich durch die proportionalen Werte, die für jede einzelne Wellenlänge des (maximal wahrnehmbaren) Spektrums das Verhältnis von einfallendem und reflektiertem Licht angeben. Aufgrund dieser Reflektanzprofile rufen undurchsichtige Körper in uns kausal Farbwahrnehmungen hervor. Reflektanztypen sind dagegen spezies-relative Klassifikationen von solchen Profilen, die mit unseren phänomenal individuierten Farbwahrnehmungstypen unter (den hier zu bestimmenden) Nor-

14 Die mitrepräsentierten Form- und Ortsaspekte werden im folgenden ignoriert werden. Hierfür werden sich jedoch ähnliche Normalbedingungen angeben lassen.

15 Generelle Schwierigkeiten für eine externalistische Gehaltstheorie, Normalbedingungen zu formulieren, werden hier nicht besprochen (vgl. Boghossian (1991); sowie die Aufsätze in Stich \& Warfield (1994)). Eine teleologisch orientierte Theorie scheint in dieser Hinsicht recht vielversprechend zu sein (vgl. Dretske (1986); (1994a); Millikan (1984); (1994)). 
malbedingungen nomologisch korreliert sind. Beide Eigenschaftsarten werden im Objektivismuskapitel noch ausführlicher behandelt. Darüberhinaus sehen wir auch durchsichtige Körper und Lichtquellen als farbig. Erstere zeigen statt einem Reflektanzvermögen ein Transmittanzvermögen (das Verhältnis zwischen einfallendem und durchgelassenen Licht pro Wellenlänge); da beide im Prinzip sehr ähnlich beschaffen sind und zudem über das Phänomen der Absorption sehr eng miteinander zusammenhängen (alles nicht-absorbierte Licht wird entweder reflektiert oder durchgelassen), können Transmittanzeigenschaften im weiteren Verlauf der Diskussion vernachlässigt werden.

Werden dagegen Lichtquellen als farbig gesehen, liegt ein Fall von Farbillusion vor (sehr ähnlich den weiter unten erwähnten Situationen mit void viewing conditions, in denen ein einzelner, farbiger Gegenstand vor ganz schwarzem, Licht nicht reflektierendem Hintergrund gesehen wird). Bis auf wenige Ausnahmen (auf die ich zum Ende dieses Abschnittes hin zu sprechen kommen werde) ist es nun zudem allgemein üblich, bei diese Detektion der Reflektanzeigenschaften eher als eine Repräsentation aufzufassen, die einerseits die Möglichkeit von Fehlrepräsentationen erlaubt, andererseits aber davon ausgeht, daß im Regelfall - eben unter Normalbedingungen - die entsprechenden Sinneserfahrungen repräsentational veridisch sind. Es ist deshalb sinnvoll, bei der Besprechung von Normalbedingungen mit der Repräsentation von diesen Reflektanzeigenschaften zu beginnen.

\subsubsection{Normalbedingungen der Repräsentation von Reflektan- zeigenschaften}

Eine ganz allgemeine,biologische Funktion von Wahrnehmungen ist es, Informationen über Tatsachen in der Welt zu erhalten. Damit eng verbunden ist die Fähigkeit, Gegenstände in der Welt aufgrund bestimmter Eigenschaften, die sie instantiieren, zu erkennen, zu diskriminieren und schließlich $\mathrm{zu}$ individuieren. Es werden also sowohl die Existenz von numerisch verschiedenen Objekten als auch deren Gemeinsamkeiten und Unterschiede erfaßt, so daß schließlich ein Bezugnehmen auf und Wiedererkennen von Einzeldingen möglich wird (vgl. Thompson (1995): 180). Visuelle Wahrnehmungen haben nun die konkretisierte Funktion, Objekte aufgrund 
ihrer sichtbaren Eigenschaften im Raum zu lokalisieren und zu individuieren. $\mathrm{Zu}$ den sichtbaren Merkmalen von Gegenständen gehören, nach einem ganz allgemeinen Verständnis, Form- und Ortseigenschaften, aber auch Distanzen und eben Reflektanzvermögen (welche wir als Farben sehen). Die besondere Rolle der Repräsentation von Reflektanzeigenschaften ist es dabei, Objekte gegen ihre Umgebung abzusetzen oder von einem Hintergrund zu unterscheiden (wie etwa reife Früchte an einem Baum zu erkennen) - und zwar vor allem unter ständig wechselnden und teilweise auch ungünstigen Lichtbedingungen. Es ist evolutionär vorteihaft gewesen, daß sich bei den meisten Lebewesen ein visueller Sinn entwickelt hat, welcher Informationen über die in der Welt instantiierten Reflektanzeigenschaften bereitstellen kann.

„Color vision has evolved to enhance wavelength differences between the reflectance of an object and its background in an environment where gradients of energy are often minimal. Color vision is not concerned with analyzing the wavelength composition of light reflected from an object's surface but with exposing an object in its background. [...] The colors we see in objects are those that best set them off from their backgrounds unter the prevailing light conditions." (Gouras \& Zrenner (1981): 139f.)

Diejenigen Eigenschaften, die weitestgehend diese Beleuchtungsunabhängigkeit zeigen und zudem sichtbar sein können, da sie lichtbeeinflußend wirken, sind die Reflektanzeigenschaften von Objekten. Sie können somit als visuell wahrnehmbares Unterscheidungskriterien für die Individuation von Gegenständen genutzt werden. Aufgrund der anatomischen und funktionalen Ähnlichkeit zwischen den visuellen Systemen verschiedener Lebewesen kann neben der weitreichenden Intersubjektivität hinsichtlich der diskriminatorischen Fähigkeiten innerhalb einer Spezies auch eine annähernde zwischen den einzelnen Spezies untereinander angenommen werden; allein verschiedene Überlebensstrategien und -bedürfnisse haben zu größeren Differenzen geführt (so daß Bienen etwa auch Licht außerhalb unseres wahrnehmbaren Spektrumes sehen können, um Blüten besser voneinander unterscheiden zu können).

Die zwischen den Menschen herrschende Intersubjektivität wird durch das Phänomen der Farbkonstanz unterstützt, welches einfach in dem Um- 
stand besteht, daß Gegenstände auch unter wechselnden, zum Teil sich erheblich verändernden Lichtbedingungen für uns immer dieselbe Farbe zu besitzen scheinen. Farbkonstanz erst ermöglicht verläßliche Wahrnehmungen von Reflektanzeigenschaften. Besteht dagegen keine Farbkonstanz, erreicht das Auge nicht mehr die erforderliche Informationen, um auf die tatsächlichen Reflektanzen schließen zu können, weil die Lichtbedingungen sich zu stark vom evolutionär bedeutsamen Normalfall unterscheiden. Es ist oft genug davon die Rede, daß es sich dann um eine Abweichung von der ,idealen“ oder ,perfekten“ Farbkonstanz handelt, die nur in den normalen Wahrnehmungssituationen, an die unser Auge sich ursprünglich angepaßt hat, besteht:

„Such departures from perfect color constancy with changes in spectral quality of illumination ... imply that perceived contrast between objects of different surface reflectance varies with the level and kind of illumination in which they are seen and to which the visual system is adapted.“ (Jameson \& Hurvich (1989): 7)

Es scheint also sinnvoll zu sein, die Bedingungen, unter denen Farbkonstanz auftritt, als Normalbedingungen für die Farbwahrnehmungen anzunehmen (vgl. auch Zeki (1993): 100). Denn nur das Vorkommen von Farb konstanz kann als Garatie für repräsentational-veridische Wahrnehmungen von Reflektanzeigenschaften angesehen werden. Doch die Bestimmung der Bedingungen, unter denen Farbkonstanz herrscht, ist nicht so einfach möglich. Es lassen sich zwar sogenannte Optimalbedingungen angeben, unter denen es generell möglich ist, veridische Farbwahrnehmungen zu haben. Aber je nach betrachtetem Objekt kann dies auch unter anderen, nicht-optimalen Umständen der Fall sein. Als Beispiel können solche Objekte dienen, die nur in einem sehr eng begrenzten Bereich des Spektrums Licht reflektieren. Für die Veridizität der Wahrnehmung solcher Reflektanzeigenschaften kann es also gleichgültig sein, welche Lichtbedingungen hinsichtlich der anderen Abschnitte des wahrnehmbaren Spektrums herrschen. Nehmen wir einen Gegenstand $x_{1}$, der nur Licht zwischen $450 \mathrm{~nm}$ und 500 $\mathrm{nm}$ zurücksendet. Im Extremfall kann beispielsweise nur Licht in diesem Bereich vorhanden sein, und dennoch kann das Reflektanzvermögen des Gegenstandes vollständig durch Sinneserfahrungen erfaßt werden, da die dazu benötigte Information allein in diesem Bereich des Spektrums vermit- 
telt wird.

Doch auch hier kann argumentiert werden, daß das Auge unter nichtoptimalen Bedingungen im Grunde das Reflektanzvermögen des fraglichen Objektes nicht korrekt erfaßt; und daß, obwohl Farbkonstanz vorliegt. Denn dem visuellen System ist trotz allem nicht die Information über das Reflektanzevermögen des Gegenstandes hinsichtlich des gesamten wahrnehmbaren Spektrums gegeben. Daß unser Gehirn in diesem Fall die fehlenden Daten der Wirklichkeit entsprechend ergänzt, ist reiner Zufall. Um daß zu sehen, genügt es, sich ein zweites Objekt $x_{2}$ vorzustellen, welches in dem Bereich zwischen $450 \mathrm{~nm}$ und $500 \mathrm{~nm}$ dasselbe Reflektanzvermögen aufweist wie der erste Gegenstand $x_{1}$, dafür aber zusätzlich noch bei 600 $\mathrm{nm}$ Licht reflektiert. Unter den meisten Lichtbedingungen werden die beiden Objekte gleich aussehen, insbesondere, wenn die Lichquelle nur Licht im Bereich von $450 \mathrm{~nm}$ und $500 \mathrm{~nm}$ aussendet. Da dies jedoch unter optimalem, kontinuierlich über das gesamte wahrnehmbare Spektrum verteiltem Licht nicht mehr der Fall ist, wird man darauf schließen, daß die Reflektanz von $x_{2}$ unter der eingeschränkten Beleuchtung fehlrepräsentiert wird, weil fehlende Information nicht richtig ergänzt worden ist.

Dies legt aber nahe, daß jede Ergänzung von Information durch das Gehirn, selbst wenn sie zufälligerweise (wie bei $x_{1}$ ) zutreffend ist, als eine partielle Fehlrepräsentation aufzufassen ist. Daß das Gehirn in beiden Fällen unter eingeschränkter Beleuchtung Information automatisch ergänzt, heißt dabei nichts anderes, als daß es die betreffenden Gegenstände als solche ansieht, die außerhalb des fraglichen Bereiches das Reflektanzvermögen „Null“ haben. Doch diese Ergänzung ist beidesmal nicht dadurch bedingt, daß der Gegenstand wirklich in diesem Bereich alles auf ihn einfallende Licht absorbiert, sondern darauf, daß in diesem Bereich gar keine Lichtwellen auf ihn treffen. Deswegen wird auch $x_{1}$ fehlrepräsentiert.

Die Begründung hierfür findet sich in den Überlegungen, welche Lewis mit Hinsicht auf die ,veridischen Halluzinationen“ angestellt hat. Denn nicht das zufällige Ergänzen von Informationen oder Hervorrufen von mentalen Zuständen, sondern nur kontrafaktische Abhängigkeit von der wahrgenommenen Szenerie kann eine Repräsentationsrelation fundieren. Danach muß zwischen repräsentierter Eigenschaft und repräsentierendem Wahrnehmungstypus eine nomologische Korrelation bestehen. Dies kann aber unter den eingeschränkten Lichtbedingungen nicht der Fall sein, wie 
der folgende Gedankengang zeigt. Nehmen wir an, $x_{1}$ hat die Reflektanzeigenschaft $S S R_{1}$ und $x_{2}$ die Reflektanzeigenschaft $R_{2}$ (gleich, ob es sich um Profile oder Typen derselben handelt). Unter den eingeschränkten Bedingungen sehen beide Gegenstände gleich aus. Wenn also in diesem Fall eine nomologische Korrelation bestehen sollte, müßten $S S R_{1}$ und $S S R_{2}$ zu einer einzigen Eigenschaft zusammengenommen werden, mit der der entsprechende Typus von Sinneserfahrungen repräsentational verbunden wäre. Dies ist jedoch nicht möglich, da unter Optimalbedingungen die beiden Gegenstände verschieden aussehen und - geht man davon aus, daß unter Optimalbedingungen Reflektanztypen korrekt wahrgenommen werden somit auch verschiedene Eigenschaften aufweisen, die mit unterschiedlichen Wahrnehmungstypen nomologisch korreliert sind.

Damit kontrafaktische Abhängigkeit von der vor den Augen befindlichen Szenerie bestehen kann, können also nur eine der beiden Lichtbedingungen als Veridizität garantierende Lichtbedingungen gelten: entweder die eingeschränkten, oder die optimalen. Betrachtet man die Funktionsweise des visuellen Systemes sowie das Phänomen der Farbkonstanz, so ist es klar, daß letztere - und nur letztere - als Normalbedingungen gewählt werden sollten. Wenn man so will und Lewis Sprechweise folgen möchte, handelt es sich bei der Wahrnehmung von $x_{1}$ unter einer Beleuchtung, welche nur aus Lichwellen zwischen $450 \mathrm{~nm}$ und $500 \mathrm{~nm}$ besteht, um eine ,veridische Halluzination“. Sieht ein Gegenstand genauso aus, wie er unter Normalbedingungen aussehen würde, reicht dies noch nicht allein aus, um von einer veridischen Wahrnehmung zu sprechen: die Veridizität kann zwar zufälligerweise Bestand haben, doch ein Teil der für die Wahrnehmung verantwortlichen Information ist nicht wahrgenommen worden, wendet man Lewis Kriterium für Wahrgenommenwerden an. So kann der Betrachter unter eingeschränkten Lichtbedingungen nur die Reflektanzeigenschaften von Objekten (ganz gleich, von welchen) innerhalb des Bereiches von 450 $\mathrm{nm}$ und $500 \mathrm{~nm}$ sehen; außerhalb davon sieht er jedoch keine Reflektanzen mehr. Hierin liegt auch der einzige Unterschied zu Lewis Beispiel: während das Reflektanzvermögen von $x_{1}$ wenigstens noch teilweise wahrgenommen wird, sieht der Mann mit dem Lichtmesser die Beschaffenheit der Landschaft im ganzen nicht.

Das heißt aber, daß Farbkonstanz allein noch nicht als Maßstab für Normalbedingungen und die Veridizität von Wahrnehmungen gelten kann; 
denn es werden auch „,veridische Halluzinationen“ der Farbkonstanz gerecht: wie im Beispiel des Objektes $x_{1}$ unter eingeschränkter Beleuchtung. Vielmehr können nur Optimalbedingungen (ohne solche Einschränkungen der Beleuchtung) als Normalbedingungen gelten. Um dieser Konsequenz nun zu entgehen, könnte man jedoch einwenden, daß unter dieser Voraussetzung viele unserer alltäglichen Farbwahrnehmungen partiell gar keine genuinen Wahrnehmungen wären (sondern „,veridische Halluzinationen“), weil nur recht selten Optimalbedingungen vorlägen; doch eine solche, häufige Fehlerhaftigkeit ist introspektiv gar nicht ersichtlich (die Ergänzungen des Gehirnes, wie etwa hinsichtlich des Blinden Fleckes, passieren für uns unbemerkt) und zeigt offensichtlich auch keine Folgen für das Funktionieren des visuellen Systemes. Doch dieser Einwand ist nicht berechtigt. Daß eine Sinneserfahrung zum Teil auf wahrgenommener und zum Teil auf ergänzter Information beruht, muß natürlich nicht ein besonderes, introspektierbares Merkmal mit sich bringen: auch vollständige Halluzinationen sind nicht von veridischen Wahrnehmungen zu unterscheiden. Daneben entspricht die Beleuchtung in den allermeisten Fällen den Optimalbedingungen (auch oftmals bei künstlichem Licht). Doch selbst, wenn dies nicht der Fall sein sollte, handelt es sich nur um eine Abweichung vom Normalfall, also um eine partielle Illusion, und noch nicht unbedingt um eine (,,veridische“) Halluzination.

Hierauf könnte man antworten, daß es doch vielleicht zu der Funktion des visuellen Systemes gehören könnte, wenigstens bis zu einem gewissen Grad fehlende Informationen zu ergänzen (wie ebem beim Blinden Fleck). Doch auch diese Strategie ist wahrscheinlich nicht erfolgreich. Zuerst einmal ist es plausibel, auch den Blinden Fleck als eine Illusion aufzufassen. Dann kann es durchaus möglich sein, daß einige Illusionen sogar evolutionär vorteilhaft sein können: das bringt zwar natürlich Probleme für die Bestimmung von Normalbedingungen mit sich, erlaubt aber trotzdem, auch in diesen Fällen von Illusionen zu sprechen. Und schließlich hat sich das visuelle System aller Voraussicht nach unter solchen Bedingungen entwickelt, die den Optimalbedingungen entsprechen: im normalen, kontinuierlichen Sonnenlicht (im Bereich von etwa $300 \mathrm{~nm}$ bis $800 \mathrm{~nm}$ ) an Orten mit einem über das Jahr relativ gleichmäßigen Licht. Unsere Augen sind dagegen sicherlich nicht besonders gut für künstliches Licht oder entsprechende Filter geeignet. Situationen mit dieser Beleuchtungsart können also 
nicht unbedingt zu den Normalbedingungen gerechnet werden (sie zeigen höchstens, wie sehr der Mensch in seine gewohnte Umgebung einzugreifen vermag). Selbst Feuer ist in diesem Sinne ein „künstliches“ Licht. Doch auch wenn man von diesen Überlegungen nicht überzeugt worden ist, kann man trotzdem von den Optimalbedingungen als dem Regelfall für Normalbedingungen ausgehen.

Deswegen stellen die in der Colorimetrie für die Farbwahrnehmungen herausgearbeiteten und sowohl hinsichtlich der Lichtbedingungen als auch der Wahrnehmungskontexte formulierten Optimalbedingungen auf alle Fälle den ersten Bestandteil der Normalbedingungen für Farbwahrnehmungen dar (vgl. Zeki (1993): Kap. 23). Für das Licht müssen folgende zwei Bedingungen erfüllt sein. Erstens muß es im Bereich des maximal wahrnehmbaren oder maximal für Farberkennung relevanten Bereiches des Spektrums kontinuierlich sein. Der maximal wahrnehmbare Bereich liegt bei den Lebewesen auf der Erde zwischen $300 \mathrm{~nm}$ und $800 \mathrm{~nm}$ : in diesem Bereich finden die meisten Interaktionen zwischen Materie und Lichtwellen statt. Beschränkt man sich auf Menschen, kann er unserem wahrnehmbaren Spektrum entsprechend eingeschränkt werden. Und zweitens müssen die Intensitäten der Wellenlängen in diesem Bereich über dem Schwellenwert für die Reizung der für Farbensehen verantwortlichen Rezeptoren liegen; zudem sollte die Energieverteilung innerhalb des Abschnittes möglichst gleichmäßig sein, so daß durch stark monochromatisches Licht verursachte Farbverschiebungen (wie beim Helson-Judd-Effekt) vermieden werden können. Für den Wahrnehmungskontext ergibt sich nur die Forderung, daß mehrere Objekte mit verschiedenen Reflektanzen gleichzeitig betrachtet werden; wird nur ein einzelnes Objekt vor schwarzem Hintergrund betrachtet (das heißt, unter void viewing conditions), kommt es $\mathrm{zu}$ Fehlrepräsentationen der Reflektanzeigenschaften. Den Simultankontrast dabei als Illusion zu beschreiben, dürfte unmöglich sein (wie weiter unten erläutert werden soll), so daß alle möglichen Konfigurationen des Kontextes (das heißt, alle möglichen Formationen der wahrgenommenen Farbträger) zugelassen sind.

Damit jedoch eine veridische Repräsentation der Reflektanzeigenschaften möglich wird, müssen noch weitere Bedingungen erfüllt sein. Das Auge (oder allgemeiner: das visuelle Sinnesorgan) des betrachtenden Subjektes muß in Richtung des wahrzunehmenden Objektes gerichtet sein, da- 
mit überhaupt das vom Gegenstand reflektierte Licht auf die Rezeptoren im Auge auftreffen kann. Die Distanz zwischen Sinnesorgan und Objekt (oder auch der Sehwinkel, unter dem das Objekt wahrgenommen wird) darf weder zu groß, noch zu klein sein; sowohl die Größe des wahrzunehmenden (Teiles eines) Gegenstandes als auch die Größe der Augenlinse werden - wenn auch relativ weite - Grenzen setzen. Zudem darf die freie Sicht nicht behindert sein: weder durch lichtundurchlässige, noch durch lichtdurchlässige, aber das Licht beeinflußende Objekte. Das vom wahrzunehmenden Gegenstand ausgesandte Licht muß das Auge möglichst ungestört erreichen können; es sollte auf dem Weg, den es zurücklegen muß, nicht mit Materie in Wechselwirkungen treten (eine Ausnahme könnten vielleicht Beispiel wie eine durch die Gravitation bedingte Krümmung der Bahn des Lichtes darstellen, wie es etwa bei Sternen der Fall sein kann). Das betrachtete Objekt darf auch seine Reflektanzeigenschaften während des Wahrnehmungsaktes nicht - oder nur derart - verändern, daß die hervorgerufene Sinneserfahrung davon nicht beeinflußt wird; da eine Wahrnehmung sich bereits auf den Bruchteil einer Sekunde beschränken kann, sollte die Erfüllung dieser letzten Bedingung kein Problem darstellen. Schließlich darf die Möglichkeit einer der Wahrnehmung zugrundeliegenden, singulären Kausalrelation zwischen dem wahrgenommenen Gegenstandes und der auftretenden Wahrnehmung nicht bereits von vorneherein durch die bestehenden, äußeren Umstände zunichte gemacht werden. Damit sind die externen Normalbedingungen bestimmt, die in der Umgebung herrschen müssen, damit Farbwahrnehmungen veridisch sein können. ${ }^{16}$

16 In Texten, die nicht auf Reflektanzen Bezug nehmen, sondern ganz allgemein von in der Welt repräsentierten Eigenschaften sprechen, finden sich dementsprechend allgemeinere Bestimmungen von Normalbedingungen. Averill und Wright schlagen zum Beispiel eine ähnliche Vorgehensweise vor, wie er im Fall der internen Normalbedingungen angewendet werden wird, indem sie die externen Normalbedingungen statistisch und auf die aktuale Welt bezogen bestimmt sehen möchten (vgl. Wright (1988): 15f.; (1992): 112ff.). Doch die Vorgaben durch die Wissenschaften der Farben erlauben zum großen Teil eine qualitative Spezifizierung der Normalbedingungen; nur hinsichtlich besonderer, quantitativer Faktoren (wie etwa der für das Farbensehen minimal erforderlichen Lichtintensität) werden statistische Meßexperimente zur Bestimmung der typischen Werte erforderlich sein. 
Doch für jedes farbwahrnehmende Subjekt müssen natürlich auch bestimmte interne Normalbedingungen erfüllt sein. Eine erste, sehr einfache Forderung ist die, daß das Subjekt aufgrund seiner Konstitution überhaupt in der Lage sein muß, phänomenale Farbwahrnehmungen haben zu können. Es wird sich eventuell nicht in allen Fällen allein anhand der wissenschaftenlichen Evidenzen über Verhalten und neurophysiologische Beschaffenheit eindeutig entscheiden lassen, ob bestimmte Tiere Farben sehen oder nicht; und andere objektive Evidenzen stehen nicht zur Verfügung. Aber im Prinzip scheint der Fall bei vielen, höherentwickelten Säugetieren (wie etwa Primaten) klar zu sein, auch deswegen, da es zu vermuten ist, daß das menschliche Farbensehen im Vergleich zu dem vieler anderer Lebewesen relativ einfach und unpräzise beschaffen ist. Somit sollten Menschen nicht als die einzigen phänomenal bewußten, farbwahrnehmenden Subjekte gelten. Aber das Vorliegen einer Wahrnehmung allein genügt noch nicht. Diese muß zudem auf eine verläßliche Weise hervorgerufen worden sein, um die Umwelt korrekt repräsentieren $\mathrm{zu}$ können. Unter welchen Bedingungen dies der Fall ist, wird aller Voraussicht nach nicht für jedes einzelne Individuum einzeln, sondern gleich relativ $\mathrm{zu}$ einer Spezies bestimmt werden können. So werden etwa für alle Menschen faktisch dieselben Faktoren dafür maßgebend sein, daß Mitglieder dieser Spezies als normalsichtige Subjekte gelten.

Wie kann aber mit Bezug auf eine Gruppe von gleichartigen Lebewesen festgestellt werden, ob und wann der Wahrnehmungsapparatus von der Reizung der Rezeptoren an bis zum Auftreten der Farbwahrnehmung hinsichtlich seiner Funktion, Reflektanzunterschiede an Objekten in der Welt zu detektieren, angemessen arbeitet? Eine Antwort können nur empirische Untersuchungen ergeben. Statistische Erhebungen über typischerweise als normalsichtig betrachtete Subjekte werden eine genauere Spezifizierung der internen Normalbedingungen ermöglichen. Zwei Schritte werden dabei erforderlich sein: der qualitative beschreibt die grundsätzlichen Anforderungen an die zu betrachtenden Subjekte; der quantitative wird diese Angaben dann konkretisieren. Ein normalsichtiger Mensch wird, aufgrund unserer neuropyhsiologischen Erkenntnisse, zum Beispiel zum einen über zwei Augen mit drei verschiedenen, chromatisch sensitiven Rezeptorarten (den Zäpfchen), und zum anderen über zwei von den Augen wegführende, optische Nerven und einen sich anschließenden, visuellen Kortex verfügen 
müssen, wobei der neuronale Teil des visuellen Systemes zusätzlich ein Gegenfarbsystem realisieren muß (letzteres bietet unter anderem eine funktionale Beschreibung davon, welche Output-Zustände des visuellen Systemes zu welchen Farbwahrnehmungen führen). Doch in welchem Bereich die Rezeptorarten für Lichtwellen in etwa sensitiv sein müssen, und welche quantitative, algorithmische Ausformulierung der funktionalen Gegenfarbtheorie einigermaßen zutreffend ist, wird sich nur statistisch über Experimente mit geeigneten Probanten bestimmen lassen.

\subsubsection{Normalbedingungen und Farbtheorien}

Nicht jede Theorie der Farben nimmt an, daß diese von Farbwahrnehmungen repräsentiert werden. Doch wenn eine Position - wie der Objektivismus - Farben als repräsentierte Eigenschaften ansieht, wird sie diese in einen engen Zusammenhang mit den Reflektanzvermögen bringen müssen: entweder durch die Identitäts- oder die Realisierungsrelation. In beiden Fällen können die Normalbedingungen von der Repräsentation der Reflektanzeigenschaften übernommen werden. Wahrnehmungen von Farben werden also dieselben Normalbedingungen wie Wahrnehmungen von Reflektanzvermögen haben. Ein subjektivistischer Dispositionalismus, der die Repräsentiertheit von Farben aufrechterhalten möchte, wird folglich ebenso aller Wahrscheinlichkeit nach die Normalbedingungen für die Repräsentationen von Reflektanzen übernehmen. Dabei kann diese Übernahme jedoch für die subjektivistischen und auch einige objektivistische Positionen problematisch werden, was jedoch erst im Zusammenhang mit den entsprechenden Theorien behandelt werden soll. Anders sieht es natürlich für Theorien aus, die die Repräsentationalität von Farben freiwillig ablehnen oder, was wahrscheinlicher ist, ablehnen müssen. Für manche Theoretiker detektieren Farbwahrnehmungen von vorneherein zwar Farben in der Welt (als Reflektanzeigenschaften verstanden), doch handelt es sich dabei nicht um einen repräsentationale, sondern nur um eine informationale Relation zur Welt. Demgemäß können diese nie falsch sein - wenn sie auftreten; und auf Normalbedingungen kann voraussichtlich verzichtet werden (vgl. Averill (1982); Tolliver (1994)).

Die Projektivisten (und Eliminativisten) leugnen dagegen nicht die Re- 
präsentationalität von Farbwahrnehmungen, sondern die Repräsentiertheit von Farben. Auch manche Vertreter eines subjektivistischen Dispositionalismus gehen diesen Weg (wie vielleicht McGinn (1983): 11). Danach werden nur Reflektanzen repräsentiert; und diese sind keine Farben. Für sie kann es deswegen natürlich hinsichtlich von Farben keine Normalbedingungen geben. Farben sind sozusagen immer notwendigerweise mit den entsprechenden Farbwahrnehmungen verbunden. Für die entsprechende Variante des Dispositionalismus sind somit alle auf Sinneserfahrungen basierenden Farburteile „korrekt“ (im Sinne eines anti-realistischen Wahrheitsbegriffes). Diese „Richtigkeit“ der Zuschreibung von Farbeigenschaften wird dabei allein anhand des Aktes des Zuschreibens selbst bewertet: bei den „Wahrmachern“ handelt es sich also nicht nur um ausschließlich auf Subjekte bezogene Tatsachen, sondern die „Richtigkeit“ hat auch einen trivialen Charakter. Stattdessen geht der Projektivismus von einem systematischen Fehler aus: zwar können die wahrgenommenen Reflektanzeigenschaften veridisch repräsentiert werden, aber die auf diese projezierte Farbigkeit entspricht nicht der Realität. In jedem Fall - mit Ausnahme der objektivistischen, informationalen Theorien über Farbdetektion - werden die im vorhergehenden Abschnitt charakterisierten Normalbedingungen übernommen: entweder nur für die Repräsentation von Reflektanzeigenschaften, oder auch für die Repräsentation von den auf ersteren zumindest supervenierenden - Farben.

\subsubsection{Die Unbestimmtheit der Wahrnehmung}

Für alle Theorien der Farben, die von Normalbedingungen ausgehen, ergibt sich das folgende, von Hardin beschriebene Problem (vgl. Hardin (1988): 67ff.). Hardin selbst wendet dieses Argument nur gegen Theorien an, die Farben als unter Normalbedingungen repräsentierte Eigenschaften ansehen. Doch wie es sich zeigen wird, thematisiert der Einwand ein generelles Problem der sinnlichen Repräsentation von Eigenschaften, welches sich sowohl auf primäre als auch auf sekundäre Qualitäten bezieht. Deshalb gilt es auch für alle Theorien, die Farbwahrnehmungen als repräsentational ansehen, ganz gleich, ob es sich bei den repräsentierten Eigenschaften um Farben oder nur um Reflektanzeigenschaften handelt. Im folgenden wird 
das Problem jedoch zuerst einmal nur anhand des Beispieles von Farbrepräsentationen erläutert. Selbst unter herrschenden Normalbedingungen weichen die Farbwahrnehmungen von Subjekten der gleichen Spezies voneinander ab; oder jedenfalls ist dies zumindest bei Menschen der Fall. Besonders auffällig werden diese Abweichungen bei der Bestimmung der vier reinen Farbtöne. Werden verschiedenen Personen zum Beispiel eine große Anzahl von in vielfältigen Grüntönen gefärbten Täfelchen vorgelegt, dann werden zum Teil sehr unterschiedliche Täfelchen herausgegriffen, wenn es um die Frage geht, welches Farbmuster einem reinen Grünton am nächsten kommt. Experimente haben gezeigt, daß die entsprechenden Positionierungen im wahrnehmbaren Spektrum in etwa den Bereich von $490 \mathrm{~nm}$ bis 520 $\mathrm{nm}$ abdecken (vgl. Hardin (1988): 79). Die Objekte, die einige Menschen als rein grün empfinden, sehen andere als grüngelb oder blaugrün - und das, obwohl jedesmal Normalbedingungen vorgelegen haben.

Die Frage ist nun, wie man die Veridizität der einzelnen Farbwahrnehmungen einstuft. Laut obigen Überlegungen müßten sie alle korrekt sein. Dann gäbe es aber Gegenstände, die gleichzeitig verschiedene Farbtöne aufweisen. Für einen subjektivistischen Dispositionalismus, der Farben als repräsentierte Eigenschaften ansieht, würde dies unproblematisch, sobald er Farben individuen-relativ bestimmen würde. Doch dann ist im Grunde der ganze Repräsentationalitätsgedanke aufgegeben worden, der ja wenigstens noch eine Restintersubjektivität retten sollte (und wie könnte jedes Individuum einer Spezies eine eigene, evolutionär begründete Repräsentationsfunktion für sein Farbwahrnehmungssystem aufweisen?). Diese Position kann also auch nicht ohne weiteres der durch Hardins Beispiel aufgeworfenen Schwierigkeit entgehen, will sie die Repräsentierbarkeit der Farben beibehalten. Ebenfalls ist es für eine Theorie der Farben nicht möglich, die Normalbedingungen einfach als genau diejenigen zu stipulieren, die repräsentationale Veridizität garantieren. Denn damit wäre man wieder am Anfang angelangt und würde sich immer noch nach der konkreten Beschaffenheit der Normalbedingungen für Farbwahrnehmungen fragen

Um diese triviale Stipulation zu vermeiden, stellt Wright die Forderung auf, daß diese Bedingungen substantiell bestimmt werden (vgl. Wright (1992): 112). Dies ist oben bereits geschehen. Die dort erfolgte Charakterisierung hat jedoch den großen Nachteil, daß sie quantitative Elemente enthält, die statistisch erfaßt werden müssen. Erst dadurch können die von 
Hardin beschriebenen Abweichungen überhaupt auftreten. Denn wie bei den meisten, statistischen Bestimmungen sind in einem gewissen Umfang Abweichungen vom Durchschnittswert zu erwarten. Eine andere Lösung, dieses Problem zu umgehen, könnte die Stipulation von sogenannten Standardbedingungen sein, die nur ganz wenige Wahrnehmungsbedingungen als normale zulassen: indem man entweder sich wirklich auf den exakten Durchschnittswert beschränkt, oder indem ganz genau festgelegte Laborbedingungen als solche stipuliert werden. Standardbedingungen vermeiden zum Beispiel Faktoren wie Beleuchtungswechsel, Schatten, Kontrastphänomene, Inhomogenitäten von Farbflächen und auch durch Materialunterschiede (wie Textur oder Glanz) bedingte Veränderungen in der Erscheinung, die zwar die Farbigkeit eines Gegenstandes nicht unbedingt verändern, aber dessen Erkennung erschweren können (vgl. Hardin (1988): 183). Doch auch diese Vorschläge führen zu nichts. Interessanterweise sind die meisten Laborbedingungen void viewing conditions und somit von vorneherein als unangemessen zu behandeln (vgl. Hardin (1988): 70). Aber auch ganz davon abgesehen führt der Weg der Stipulation von exakt gefaßten Normalbedingungen zu keinem zufriedenstellenden Ergebnis. Hardin kommentiert diese Auswegsmöglichkeit wie folgt:

\begin{abstract}
„There are two well-worn strategies for handling situations of this sort. The first is to specify a standard observer, analogous to the 1931 C.I.E. average observer, an average of a largish sample of actual normal observers, and decree that the color judgments of the standard observer have normative force. But the consequence of any stipulation of this sort is that very large numbers, probably a majority, of „normal“ observers would have unique hue loci at variance with those of the standard observer. One would also have to make a similar stipulation of standard illumination, standard viewing conditions, etc., with comparable divergences among observers for these other illuminations and viewing conditions. By decree, there would then be a fact of the matter as to whether a particular stimulus under particular circumstances was unique green, though most people would fail to see it in its ,true“ colors. In a similar fashion, we could also establish a fact of the matter about the intrinsic worth of paintings and innumerable other puzzling concerns.“(Hardin (1988): 89f.)
\end{abstract}

Es sollte sicherlich vermieden werden, daß die Veridizität von Sinneserfahrungen auf diese Weise durch Konventionen bestimmt wird, zudem dann 
auch die unplausible Konsequenz sich ergeben würde, daß die Mehrheit unserer Farbwahrnehmungen wahrscheinlich falsch sein würden. Beides widerspricht vor allem der Idee, daß unser visuelles System die biologische Funktion hat, Reflektanzeigenschaften zu detektieren: eine Funktion, die es anscheinend immer noch in den meisten Situationen angemessen erfüllen kann. Doch bei dem hier dargestellten Problem der intersubjektiven Variation handelt es sich um eine generelle Schwierigkeit von Wahrnehmungen. Demnach ist es genauso schwierig, Normalbedingungen für Formwahrnehmungen zu formulieren. Natürlich wird das Poblem für die Farbwahrnehmungen durch diesen Vergleich nicht gelöst; aber es wird vielleicht ein wenig seiner Überzeugungskraft beraubt: wenn es alle (wenigstens visuell) wahrnehmbaren Eigenschaften betrifft, dann eben auch primäre und sekundäre Qualitäten gleichermaßen. Und da für erstere dieses Problem meistens gar nicht thematisiert wird, kann es dementsprechend nicht so schwerwiegend sein.

Warum sollte diese Verallgemeinerung aber gelten? Der Grund ist einfach der folgende: für eine Theorie, die Farben das Merkmal der Repräsentierbarkeit zuschreiben möchte, ergeben sich die oben genannten Schwierigkeiten mit der Spezifizierung der Normalbedingungen nicht deshalb, weil es sich um Farben handelt, die repräsentiert werden, sondern, weil sie mit einem Sinnesorgan wahrgenommen werden. Wahrnehmung zeigt wesentlich eine gewisse Unbestimmtheit (vgl. hierzu Hilbert (1987): Kap. 6). Zum einen liegt das an den individuellen Unterschieden zwischen den Menschen in der physikalischen Konstitution. Es wäre sehr erstaunlich, wenn die Zäpfchen aller Menschen exakt dieselbe Sensitivität aufweisen und im visuellen Kortex die eingegangenen Reize mit derselben quantiativen Genauigkeit weiterverarbeitet würden. Zum anderen aber auch an der Charakteristik des Auges, wie ein Meßinstrument zu funktionieren und somit ein vorgegebenes „Auflösevermögen“" zu haben, welches einkommende Reize nur bis zu einer bestimmten Feingradigkeit oder Detailgenauigkeit registrieren und in Information umwandeln kann.

Es gibt natürlich offensichtliche Unterschiede zwischen Farb- und Formwahrnehmungen und den mit beiden verbundenen Unbestimmtheiten. Zuerst einmal zeigen Formen keine phänomenale Reinheit oder Gemischtheit, wie es bei Farben der Fall ist; und auch die Ähnlichkeitsstruktur von Formen ist ganz anders beschaffen als die kreisförmige der Farbtöne. 
Trotzdem lassen sich für Formen Eigenschaften zweiter Ordnung finden, mit deren Hilfe ein ähnliches Problem formuliert werden kann wie das, welches Farben aufgrund der abweichenden Zuschreibungen von Reinheit zeigen. Dabei ist es gar nicht so wichtig, welche Eigenschaften zweiter Ordnung betrachtet werden, da hier nur ihre Funktion von Interesse ist, eine Individuation der fraglichen Eigenschaften erster Ordnung zu ermöglichen. Für Farben bietet sich da Reinheit besonders an, weil diese insgesamt nur vier Farbtönen zukommt und zudem recht leicht festgestellt werden kann. Mit Bezug auf Formen ist es vielleicht etwas schwieriger, entsprechende Eigenschaften $\mathrm{zu}$ finden; aber es bieten sich auf alle Fälle die folgenden Beispiele an.

Gegeben ist eine große Menge von einfachen, sich sehr ähnelnden und gleich ausgerichteten Trapezen, die auf den ersten Blick, sagen wir, in der rechten, oberen Ecke einen rechten Winkel besitzen. Nun können verschiedene Betrachter dazu aufgefordert werden, diejenigen Formen aus dieser Menge herauszugreifen, die bei genauerer Betrachtung dort auch wirklich rechtwinklig sind. Oder vor den Betrachtern liegt eine Vielzahl von nahezu kreisrunden Objekten, die allesamt etwa von der gleichen Größe sind und aus denen die Form herausgesondert werden soll, welche am ehesten einem exakten Kreis gleicht. Und schließlich gibt es noch einfachere Fälle: aus einer Unzahl von fast geraden Linien soll genau die bestimmt werden, die einer Gerade am nächsten kommt. Daß solche Formeigenschaften wie Rechtwinkligkeit, Rundheit oder Gradlinigkeit - von uns nicht ganz exakt den Tatsachen entsprechend wahrgenommen werden können, sollte offensichtlich sein. Die Physik wird Längen und Formen immer genauer spezifizieren können als unser visueller Sinn. Es ist zudem zu erwarten, daß unsere Meinungen über diese Eigenschaften in Härtefällen ebenfalls auseinandergehen - wie in dem Beispiel hinsichtlich des reinen Grüntones. Zwar wird die Abweichung bei Formen vielleicht quantiativ nicht so stark ausfallen wie bei Farben - und dies ist auch kein Wunder, da die Farberkennung wahrscheinlich komplexer ist als die Formerkennung; aber qualitativ ist die Abweichung bei beiden zu finden.

Man könnte nun einwenden, daß es doch noch einen wesentlichen Unterschied gibt: denn während ein Objekt als exakt kreisrund (oder exakt rechtwinklig oder exakt geradlinig) gesehen werden kann, ohne es tatsächlich zu sein, scheint es doch wohl kaum möglich zu sein, sich über die 
Reinheit eines Grüntones zu täuschen. Doch in diesem Einwand stecken gleich mehrere Fehler verborgen. Zum einen kann es natürlich der Fall sein, daß man sich hinsichtlich der Reinheit eines Grüntones täuschen kann: und zwar, wenn man auf eine entsprechende Grünwahrnehmung introspektiert und die Introspektion fehlerhaft verläuft, so daß die Sinneserfahrung nicht als Wahrnehmung eines reinen Grüntones erkannt wird. Auch wenn solche Fehlrer recht selten vorzukommen scheinen, ist Introspektion trotzdem nicht infallibel. Zum anderen werden in der Erwiderung die Ebenen von Wahrnehmung und Introspektion vertauscht. Natürlich sollte es möglich sein - nimmt man den Vorschlag, Farben als repräsentierte Eigenschaften anzusehen, ernst -, daß ein Gegenstand mit einem reinen Grünton auch fehlrepräsentiert werden kann, indem er zum Beispiel als blaugrün gesehen wird. Dagegen ist es eine ganz andere Frage, ob man sich darüber irrt, gerade eine Wahrnehmung von einem Objekt mit einem reinem Grünton zu haben (ganz unabhängig davon, ob diese Sinneserfahrung veridisch ist oder nicht). Die gleiche Unterscheidung kann auch bei Formwahrnehmungen getroffen werden: die Form eines Gegenstandes kann fehlerhaft repräsentiert werden, oder aber die Individuation der Wahrnehmung hinsichtlich der repräsentierten Eigenschaft kann fehllaufen.

Das von Hardin vorgebrachte Argument ist somit gleichermaßen gegen die Wahrnehmung primärer und sekundärer Eigenschaften gerichtet. Eine Abweichung von der Realität wird bei den allermeisten Wahrnehmungen wenn nicht bei allen - vorliegen, da Wahrnehmen von sich aus eine gewisse Unbestimmtheit aufweist. Empirische Untersuchungen werden immer zu exakteren Ergebnissen kommen als unsere Sinne. Für sich genommen stellt dies auch gar kein Problem dar, da es sinnvollerweise nicht die Funktion unseres visuellen Wahrnehmungsapparates ist, eine größere Genauigkeit zu zeigen. Zwar zeigt sich mit Bezug auf Zuschreibungen von Eigenschaften zweiter Ordnung bei Farben eine quantitativ größere Abweichung als bei Formen, aber prinzipiell liegt dieselbe, qualitative Unbestimmtheit vor. Zudem besteht - vor dem Hintergrund der Möglichkeit einer erfolgreichen, objektivistischen Analyse der Farben - kein genereller Grund, die Reinheit von Farben anders zu behandeln als etwa die Geradlinigkeit von Linien, die Rechtwinkligkeit von Winkeln oder die Rundlichkeit von Formen. Denn auch Formwahrnehmungen sind wesentlich unbestimmt. Es ist aller Voraussicht sogar unmöglich, eine Form ganz exakt wahrzunehmen: 
„If a square surface is characterised as one enclosing exactly four equal angles whose four sides are exactly equal in length, then squareness simply isn't visually salient: there is, actually, no such thing as a visual system's functioning in a fashion conducive to the competent registration of squareness. It will be, experimentally, all the same to the subject whether the perceived object is square or merely approximately so.“ (Wright (1988): 17)

Besteht aber damit nicht doch ein - wenn auch ganz anderer als ursprünglich vermuteter - Unterschied zwischen Farb- und Formwahrnehmungen? Denn auf einmal ist es gar nicht mehr so klar, ob sich dasselbe von Farben sagen läßt: augenscheinlich erkennen wir doch sehr gut, wenn ein Gegenstand einen reinen Farbton instantiiert. Wenn dies bei Formwahrnehmungen nicht der Fall ist, besteht dann nicht eine Asymmetrie? In dieser Frage verbirgt sich erneut der Fehler, Realität und Wirklichkeit zu verwechseln. Wenn Quadratischsein so geometrisch definiert wird, wie es Wright beschreibt, dann können wir Quadrate nur annähernd als solche bestimmen, da wir Formen nicht auf die von der Geometrie vorgebene Weise sehen können. Dementsprechend ist unser sinnlich erworbener Begriff des Quadratischseins ganz strikt von dem geometrischen zu trennen (vgl. Peacocke (1992): 74). Uns sind Quadrate sinnlich ganz anders gegeben, als die Geometrie diese spezifiziert. Trotzdem ist es uns möglich, bei einigen Objekten aufgrund unserer Sinneserfahrungen sicher zu sein, daß es sich um Quadrate handelt - nicht im geometrischen, aber im phänomenalen Sinn: es handelt sich hier um zwei ganz verschiedenen Auffassungsweisen der einen Realität. ${ }^{17}$

Dasselbe kann sich nun auch bei Farben zeigen: der theoretisch oder wissenschaftlich und der phänomenal oder sinnlich erworbene Reinheitsbegriff können sich elementar unterscheiden. Zusätzlich kann es gut möglich sein, daß die - im Sinne des wissenschaftlichen Realismus - tatsächlich existierenden Reinheitstatsachen genau genommen nicht exakt wahrnehmbar sind: in demselben Sinne, wie es die geometrisch spezifizierten Formtatsachen nicht sind. Und trotzdem können wir ohne weiteres Objekte

17 Diese Idee spiegelt sich in der Postulierung von (je nach Theorie unterschiedlich aufgefaßten) phänomenalen oder perzeptuellen Begriffen (zusätzlich zu den sogenannten kognitiven) wider (vgl. Peacocke (1992): Kap. 3; aber auch Tye (1995): Kap. 6). 
in etwa als quadratisch oder als einen reinen Farbton besitzend sehen. Worüber man sich nun streiten kann, ist, ob dadurch nun wirklich die meisten oder sogar alle - unserer Wahrnehmungen ihre Veridizität (oder gar ihre Repräsentationalität) verlieren. Mir scheint es dabei plausibler zu sein, die Unbestimmtheit der Wahrnehmung - wie auch die Unbestimmtheit naturwissenschaftlicher Messungen - anzuerkennen, ohne dadurch die Abweichungen unserer Erscheinungen von der Realität als Fehlrepräsentationen anzuprangern (auch wenn dies im Fall der Farben möglicherweise aufgrund der Größe der Abweichungen vorstellbarer ist). Denn im großen und ganzen nähern sich unsere Wahrnehmungen den Tatsachen ausreichend an, so daß die biologische Funktion des visuellen Systemes - welche schließlich den letzten Maßstab darstellen sollte - dadurch nicht beeinträchtigt wird. ${ }^{18}$

\subsubsection{Probleme der externen Normalbedingungen}

Die Unbestimmtheit der Wahrnehmung betrifft allein die Normalsichtigkeit der wahrnehmenden Subjekte. Doch gerade auch bei der Formulierung der externen Normalbedingungen treten einige Schwierigkeiten auf. In seinem Buch Color for Philosophers (1986) führt Hardin eine ganze Reihe von Problemen auf, die im folgenden kurz betrachtet werden sollen. Zuerst einmal werden ganz verschiedene Gegenstände von uns als farbig gesehen. Selbst wenn man die selbstleuchtenden Körper beiseite läßt (da es sich bei diesen, wie die naturwissenschaftlich gewonnenen Erkenntnisse über die Farben gezeigt haben, nur bedingt um farbige Objekte handelt - vgl. Zeki (1993): 238), bleiben noch genügend Gegenstände übrig, für die es nicht klar zu sein scheint, ob die beschriebenen Normalbedingungen für die Wahrnehmungen dieser Objekte angemessen sind. Hardin führt, neben von sich aus Licht ausstrahlenden Gegenständen (wie Sterne und Neonlampen), explizit den Mond, Leuchtfische, direkt-reflektive Materialien (Körper, die Licht richtungsabhängig in verschiedenen Proportionen reflektieren), besondere Filter, den Himmel sowie Phänomene wie einen Regenbogen an (vgl. Hardin (1988): 68f.).

18 Byrne und Hilbert akzeptieren im Zweifelsfall ebenfalls eine sehr ähnliche Konsequenz (vgl. (1997c): 274). 
Die Frage, die Hardin stellt, ist nun, ob für die Wahrnehmung der Farbigkeit dieser Gegenstände wirklich einheitliche Normalbedingungen gefunden werden können. Wie er überzeugend zeigt, können Standardbedingungen - wie sie etwa in Laboratorien oder auch hinsichtlich bestimmter, konventioneller Klassifikationen von Farbparadigma innerhalb der Wissenschaften angewandt werden - diese Frage nur für jedes Objekt einzeln beantworten. Der Mond muß zum Beispiel aus einer wesentlich größeren Distanz betrachtet werden als etwa ein Insekt; und bei den direkt-reflektiven Gegenständen kann sich die gesehene Farbe sofort ändern, wenn die Position der Lichtquelle, des betrachteten Objektes oder auch des Betrachters selbst wechselt; für Phänomene wie einen Regenbogen sieht es wieder ganz anders aus (hier wird es insbesondere schwierig, von einer Größe des Objektes zu sprechen: doch ein Regenbogen ist aller Voraussicht eine Farbillusion, da er, wie etwa auch das Lichtspektrum beim Prisma, durch Lichtbrechung entsteht).

Doch zum Glück können die Normalbedingungen so allgemein formuliert werden, daß es prinzipiell kein Problem gibt, mit diesen Fällen umzugehen. Die möglichen Betrachtungsdistanzen sind ganz einfach durch den Umstand festgelegt, daß der wahrzunehmende Gegenstand auch tatsächlich wahrgenommen werden kann: er muß im Sehwinkel des Auges liegen. Auch wenn die damit erforderliche Entfernung sich im konkreten Fall sehr unterscheiden kann, genügt die allgemeine Spezifizierung über diesen Winkel. Dies gilt auch für alle anderen Faktoren der externen Normalbedingungen. Als Resultat wird sich dabei zum Beispiel ergeben, daß es von der Erde nicht möglich sein wird, die Farbe des Mondes hundertprozentig korrekt zu sehen, da das von diesem ausgestrahlte Licht immer seinen Weg durch die Atmosphäre bahnen muß und dort beeinflußt wird. Aus einem Raumschiff dagegen stellt dies kein Problem dar. Natürlich herrscht auch auf der Erdoberfläche eine Atmosphäre, aber hier sind die Effekte so vernachlässigbar gering, daß im Rahmen der Unbestimmtheit der Wahrnehmung normalerweise von einer Entsprechung der Erscheinungen mit der Realität ausgegangen werden kann. In sternenklaren Nächten kann eventuell für die Betrachtung des Mondes ähnliches gelten; und die Stärke des Mondlichtes (oder auch dem der Sonne) ist im Vergleich zur Absorptionsfähigkeit in der Atmosphäre groß genug, so daß immer einiges Licht relativ ungestört hindurchkommt. Doch gerade Lebewesen, die auch im ultravio- 
letten Bereich eine Farbempfindlichkeit zeigen, sehen die Sonne nicht so, wie sie aus dem Weltraum betrachtet aussähe, da die Atmosphäre als UVFilter dient. Teile des Himmels, der ja für diese Beeinträchtigung mitverantwortlich ist (gerade, indem er eine Farbe zeigt), können dagegen ohne weiteres unter Normalbedingungen in ihrer Volumenfarbigkeit korrekt repräsentiert werden.

Ein anderes Problem leitet sich von dem begrenzten Auflösungsvermögen her. Zum einen können makroskopische Gegenstände eine ganz andere, oder sogar weitestgehend gar keine, Farbe zeigen, sobald Teile von ihnen mikroskopisch betrachtet werden (wie es beim Blut der Fall ist). Und zum anderen können wir sogenannte pointillistische Bilder bei genügender Entfernung oder geringer Größe der einzelnen Punkte als aus Farbflächen bestehend sehen, während bei genauerer Betrachtung nur die Farbpunkte vorliegen (zum Beispiel beim Fernseher oder auch in Graphiken). Doch beide hängen, wie auch der eben erwähnte Sehwinkel, mit der Unbestimmtheit der Wahrnehmung zusammen. Als eine Art „Meßinstrument“ hat auch das Auge nur ein begrenztes Auflösungsvermögen. Dies beeinträchtigt jedoch auch die Wahrnehmungen von Formen oder Längen. Wie wir keine mikrospkopischen, sondern nur makroskopische Längen sehen können, gilt ähnliches auch für Farben: während uns die Farbigkeit der wirklich kleinen Objekte ohne weitere, optische Hilfsmittel entgeht, können wir trotzdem die Farbigkeit mittelgroßer Gegenstände (annähernd) veridisch erkennen.

Es besteht auch kein Widerspruch, wenn das makroskopische Ganze andersfarbig ist als die einzelnen, mikroskopischen Teile. Denn Vergleichbares zeigt sich ebenfalls wiederum bei Formeigenschaften. Wenn wir eine Linie unter dem Mikroskop betrachten, entspricht die Länge des gesehenen Teiles nicht mit der Gesamtlänge der Linie überein: vor allem auch, da sie um ein Vielfaches vergrößert dargestellt wird. Und zudem ist es möglich, daß die Linie unter dem Mikroskop Lücken aufweist, obwohl sie dem bloßen Auge kontinuiierlich zu sein scheint. Dieses Resultat wird sogar zu erwarten sein, wenn man mithilfe von Elektronenmikroskopen in die Nähe von Molekülen kommt. Wenn also die Farbe des Blutes von uns fehlerhaft gesehen wird, dann auch die Kontinuität dieser diskontinuierlichen „Linie“. Wieder kann man darüber disputieren, ob nun unsere gewöhnlichen Wahrnehmungen, weil sie den wissenschaftlich postulierten Tatsa- 
chen nie ganz exakt gerecht werden können, als Fehlrepräsentationen gelten sollten. Doch eine positive Antwort hierauf würde auch bedeuten, daß letztlich ebenso alle empirischen Messungen als falsch zu betrachten wären, da sie genauso wie unsere Wahrnehmungen immer eine Unbestimmtheit zeigen werden (es ist nur eine den technischen Mitteln entsprechende, beliebig genaue Annäherung möglich).

Neben dem räumlichen oder eigenschaftsbezogenen „Auflösungsvermögen" kommt dem Auges auch auch ein zeitliches zu. Die Rezeptoren weisen eine, wenn auch minimale, Trägheit in der Reizaufnahme auf, die beim Schauen von Fernseh- oder Kinofilmen überhaupt erst den Eindruck eines flackerfreien Bildes mit kontinuierlichen Bewegungen entstehen läßt. Inbesondere die letztere Kontinuität scheint ohne ein beschränktes Auflösungvermögen gar nicht erklärt werden zu können. Sähen wir geometrische Punkte in einer diskreten, absoluten Zeit (wenn es diese überhaupt gibt), dann könnten wir keine Kontinuität von Raum und Zeit und auch keine Bewegungen mehr wahrnehmen. Hardins entsprechenden Beispiele - wie die Möglichkeit, durch bestimmte Pulsation von achromatischen Bildern Farbempfindungen in Menschen hervorzurufen (vgl. Hardin (1988): 72f.) - lassen sich so ebenfalls auf die Unbestimmtheit der Wahrnehmung zurückführen. Es ist, alles in allem, somit wohl angebrachter, die Veridizität von Wahrnehmungen immer vor dem Hintergrund ihrer Unbestimmtheit zu bewerten. Unter Normalbedingungen repräsentieren Farbwahrnehmungen im Rahmen ihres „Auflösungsvermögens“ - die Eigenschaften, die sie repräsentieren (ob Farben oder Reflektanzeigenschaften), immer veridisch. 